\title{
Die Silbifizierung hoher Vokoide: das Deutsche im Sprachvergleich ${ }^{1}$
}

\begin{abstract}
Die wortinitialen Segmente in Deutsch ja, jung sowie die Zweitkomponenten in den so genannten schließenden Diphthongen wie in Hai, Heu, Hau weisen im Vergleich zu hohen Vokalen in Kuh, Knie eine stark variierende Artikulation auf - zudem treten diese Laute in unterschiedlichen Kontexten auf. Die hier beobachtbaren Zusammenhänge zwischen Distribution und Aussprache lassen auf durch unterschiedliche silbische Positionen bedingte Allophonie schließen (Morciniec 1958; Shannon 1984; ${ }^{2}$ Hall 1992; für Englisch: Jakobson/Fant/Halle 1952, S. 20). Eine solche Analyse, die zudem eine erhebliche Reduktion des Phoneminventars beinhaltet, konnte sich bislang für das Deutsche nicht durchsetzen: Gewöhnlich sind sowohl die schlieBenden Diphthonge als auch [j] im deutschen Phoneminventar aufgeführt; letzteres Segment wird sogar meist als Frikativ klassifiziert. ${ }^{3}$

Der Sprachvergleich ergibt neue phonologische Generalisierungen, die eine durch Silbenstruktur bedingte allophonische Analyse stützen. Insbesondere lassen sich Abstufungen erkennen, die auf durch Sonorität bestimmte Silbifizierungsbedingungen schließen lassen.
\end{abstract}

\section{Einleitung}

Sprachübergreifend lässt sich feststellen, dass die Klassifikation von Gleitlauten und Diphthongen oft kontrovers ist. Gleitlaute werden als Konsonanten, als Vokale, oder als Bestandteile von Diphthongen analysiert. Diphthonge werden mono- oder biphonemisch klassifiziert, wobei die Zuordnung der Bestandteile wiederum umstritten ist: Für den Diphthong in deutsch Heu etwa sind unter anderem / oi/, / or/, /øy/ und / ay/ vorgeschlagen worden.

1 Ich danke Lutz Gunkel, Marc van Oostendorp und vor allem Markus Hiller für ausführliche Kommentare und Anregungen. Hector Feliciano danke ich für seine unermüdliche Hilfe bei der Fertigstellung des Manuskripts.

2 Shannon spricht hier nicht explizit von Allophonie, sondern weist lediglich darauf hin, dass die Laute [j],[i] und [i] im Deutschen in unterschiedlichen Silbenpositionen auftreten, vgl. auch Moulton (1962).

3 Manche Autoren erkennen eine partielle Allophonie an. So analysiert Trubetzkoy (1958 [1938], S. 168 f.) das initiale Segment in [ja] $j a$ als Allophon von /i/, wertet die deutschen Diphthonge aber als monophonemisch. Becker (1998, S. 148) wertet die Diphthonge als biphonemische Sequenzen /ai/, /au/ und /oi/, äußert sich aber nicht zu prävokalischem [j]. Wurzel (1970, S. 244) analysiert prävokalisches [j] und das finale Segment in Diphthongen wie [ai] ei als durch unterschiedliche Silbenpositionen bedingte Allophone eines Phonems /j/. 
Eine mögliche Ursache für den Mangel an Konsens liegt in der besonderen Stellung der hohen Vokoide in der Sonoritätsskala in (1), wo Sprachlaute aufgrund von inhärenter, Schallfülle' (Lautheit) angeordnet sind. Nimmt man an, dass Laute um so eher im Silbenrand erscheinen, je weniger sonor sie sind, und um so eher den Nukleus bilden, je sonorer sie sind, so ergibt sich ein Zwischenbereich von Lauten, die zwar am ehesten in sämtlichen Silbenpositionen zugelassen, gleichzeitig aber auch die jeweils „schlechteste“ Besetzung sind.

$$
\begin{aligned}
& \text { optimaler Silbenrand optimaler Nukleus } \\
& \text { weniger sonor sonorer } \\
& \text { Obstruenten }>\text { Nasale }>1>\mathrm{r}>\text { hohe }>\text { mittlere }>\text { tiefe } \\
& \text { Vokale Vokale Vokale }
\end{aligned}
$$

Im Folgenden wird davon ausgegangen, dass die in (1) dargestellten Präferenzen hinsichtlich der Zuordnung von Segmenten und Silbenpositionen einzelsprachlich in Form von notwendigen Zuordnungen grammatikalisiert werden. So deutet der Umstand, dass die auf $/ \mathrm{r} /$ endenden englischen Wörter in (2a) inzwischen reimen, die auf / $1 /$ endenden Wörter aber weiterhin verschieden sind, auf eine historische Verschiebung der für Silbifizierung relevanten Sonoritätsschwellen hin.
a. $/ \mathrm{sr} / \operatorname{sir}, \mathrm{Herr}^{6}$ / fr/ fur, Fell' /hr/ her , ihr ${ }^{6}$
b. /ril/ rill, Bächlein` /ful/ full, voll' /usl/ well,Brunnenc

Die Verschiebung ist in (3a) vs. (3b) durch die Balken markiert. Die Grammatikalisierung kommt durch die Attribute „notwendig“ versus „möglich“ zum Ausdruck.

$$
\text { notwendiger Rand möglicher Nukleus }
$$

a. Obstruenten $>$ Nasale $>1>\mathrm{r} \square$ hohe $>$ mittlere $>$ tiefe Vokale Vokale Vokale

b. Obstruenten $>$ Nasale $>1 \square \mathrm{r}>$ hohe $>$ mittlere $>$ tiefe Vokale Vokale Vokale

Die Beispiele in (4) veranschaulichen den grammatischen Wandel: Nach$\mathrm{dem} / \mathrm{r} / \mathrm{auch}$ als Nukleus zugelassen war, verschwanden die historischen Vokale. ${ }^{4}$ Das Phonem /l/ hingegen ist weiterhin auf den Silbenrand beschränkt (vgl. (4b)), die Vokale werden folglich weiterhin als Nukleus benötigt $(\sigma=$ Silbe, $\mathrm{A}=$ Ansatz, $\mathrm{N}=$ Nukleus, $\mathrm{K}=\mathrm{Koda}):^{5}$

\footnotetext{
$4 \quad$ Vokale mit relativ hoher Sonorität blieben jedoch auch vor $/ \mathrm{r} /$ als Nukleus erhalten (vgl. /far/ far, /for/ for .

Aus Platzgründen sind die prosodischen Darstellungen im Folgenden auf den jeweils relevanten Bereich beschränkt.
} 
Phonemische Ebene: /s I r/

Phonetische Ebene: a.

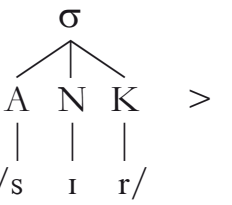

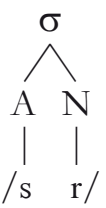

[S3: sir b.

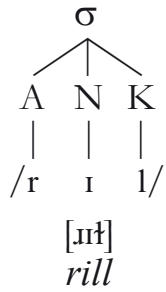

Die Darstellung des grammatischen Wandels in Form von zulässigen Verbindungen zwischen Segmenten und Silbenpositionen wie in (3) verdeutlicht die Notwendigkeit der Trennung von phonemischen und phonetischen Ebenen. So unterscheidet sich der letzte Laut im Amerkanischen Englischen wie in $\mathrm{s}[3 \mathrm{~s}]$ sir phonetisch recht stark vom initialen Laut wie in [x] ill „Bächlein‘. Die fraglichen Unterschiede lassen sich jedoch bei der Annahme eines einzigen Phonems / $r$ als Funktion der unterschiedlichen Silbenpositionen erklären: Folgt ein Phonem mit höherer Sonorität wird /r/ als Ansatz organisiert und konsonantisch realisiert (vgl. / rrl/ in (4b)); erscheint $/ \mathrm{r} /$ in einer Umgebung von weniger sonoren Segmenten, so wird es notwendig als Nukleus organisiert und silbisch gesprochen (vgl. /sr/ in (4a)).

Das Ziel der vorliegenden Studie ist es, die Verteilung unterschiedlicher Vokoide im Deutschen durch Regeln für die prosodische Organisation von Phonemen mit Bezug auf die Sonoritätsskala zu erklären. Eine wichtige Annahme betrifft dabei die feinere Ordnung der hohen Vokale in (5), derzufolge ungespannte Vokale sonorer sind als gespannte, die wiederum skaliert sind. Sprachübergreifend gilt, dass von allen Vokalen /i/ am ehesten in einer Randposition erscheinen kann, gefolgt von /u/.

$$
\begin{aligned}
& \text { optimaler Rand } \\
& \text { weniger sonor } \\
& \text { optimaler Nukleus }
\end{aligned}
$$

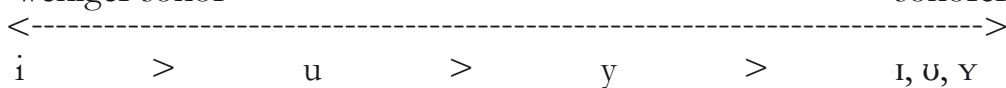

Die Annahme, dass gespannte hohe Vokale eher dem Rand zuordenbar sind als ungespannte, erklärt bestimmte Neutralisationserscheinungen im Englischen. So kontrastieren nur hohe gespannte Vokale vor Folgevokal, eine Beschränkung, die in betonten und unbetonten Silben gilt (vgl. (6a), (6b)).

$$
\begin{aligned}
& \text { a. [pí.ən] paean ,Lobrede“ } \\
& \text { [drú.Id] druid ,Druide }
\end{aligned}
$$

b. [ízri.əl] Israel [bédu.In] bedouin

Der Umstand, dass andere Vokale, einschließlich hoher ungespannter Vokale, in dieser Position ausgeschlossen sind, weist auf eine unverletzbare Beschränkung gegen Hiatus im Englischen hin. Die Folge zweier Nuklei ist demnach nicht zulässig (vgl. die ungrammatischen Strukturen in $(7 \mathrm{a}, \mathrm{b}))^{6}{ }^{6}$

Im Gegensatz zum Deutschen sind im Englischen ungespannte Vokale in offenen Silben erlaubt (z.B. b[ז.]nign , gütig). 
Dass hohe gespannte Vokale aber doch vor einem Nukleus auftreten wie in (6), lässt darauf schließen, dass diese Vokale allein zusätzlich mit dem Silbenrand assoziiert sind und somit keinen Hiatus ergeben. Diese Bedingung ist in (7c) dargestellt, wo gespanntes /i/ gleichzeitig mit Nukleus und Koda assoziiert und das Nebeneinander zweier Nuklei somit nicht gegeben ist $(\Sigma=\mathrm{Fu} \beta)$ :
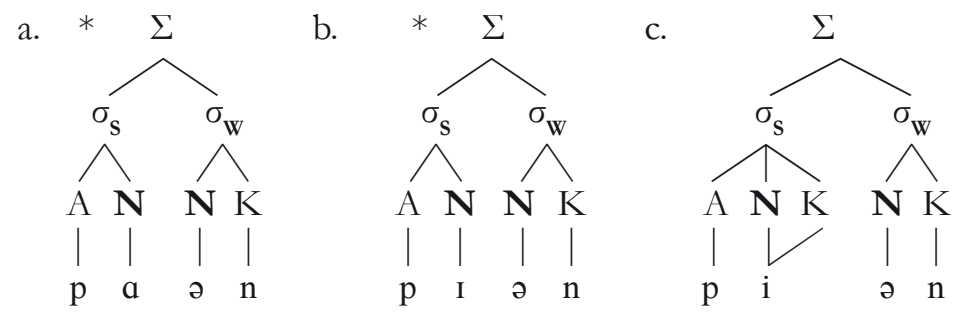

Die prosodische Darstellung des Grammatikalitätsunterschiedes in $(7 \mathrm{~b} / \mathrm{c})$ motiviert die Ordnung der gespannten versus ungespannten hohen Vokale in (5), da die Assoziierbarkeit eines Phonems mit dem Silbenrand gegenüber ausschließlicher Nukleuszuordnung auf geringere Sonorität schließen lässt.

Die Abstufungen innerhalb der hohen gespannten Vokale in (5) lassen sich durch Silbifizierungsmuster im Französischen motivieren. Die durchweg einsilbig gesprochenen Wörter in (8a) zeigen, dass ein Konsonant und der nachfolgende hohe Vokal als komplexer Ansatz organisiert werden. Die Skalierung in (5) wird durch regionale Unterschiede bestätigt, da in manchen Varianten nur /i/ und /u/ als Zweitelemente in komplexen Ansätzen erscheinen (siehe (8b)), in anderen nur /i/ (siehe (8c)) (siehe Tranel 1987, S. 122). ${ }^{7}$

\begin{tabular}{|c|c|c|}
\hline a. $[$ nie $] / *[$ ni.e $]$ nier & b. [nie]/*[ni.e] & c. $[$ nie $] / *[$ ni.e $]$ \\
\hline$[$ nue $] / *[$ nu.e $]$ nouer & [nue] $/ *[$ nu.e] & $*[$ nue $] /$ [nu.e] \\
\hline $\begin{array}{l}{[\text { nye }] / *[\text { ny.e] nuer }} \\
\text { sortieren' }\end{array}$ & $*[$ nye $] /$ [ny.e] & $*[$ nye $] /$ [ny.e] \\
\hline
\end{tabular}

Der Sprachvergleich legt daher die Plausibilität des grammatischen Wandels in (9) nahe, demzufolge / $\mathrm{u} /$ nicht mehr als Ansatz silbifiziert werden kann, diese Möglichkeit für /i/ jedoch weiter bestehen bleibt:
möglicher Ansatz unmöglicher Ansatz

$\begin{array}{llllll}< & & & \\ \text { a. } & \mathrm{i} & \mathrm{u} & \mathrm{y} & > & \mathrm{I}, \mathrm{U}, \mathrm{Y} \\ \mathrm{b} . \mathrm{i} & \mathbf{\mathrm { u }} & \mathrm{u} & \mathrm{y} & > & \mathrm{I}, \mathrm{U}, \mathrm{Y}\end{array}$

Für weitere Evidenz für die Skalierung in (5) im Französischen und Englischen siehe Raffelsiefen (eingereicht). 
In der vorliegenden Studie soll nachgewiesen werden, dass die Darstellung in (9b) die synchrone Grammatik des Deutschen abbildet, entgegen der Annahme einer parallelen Entwicklung beider hoher Vokale wie etwa bei Vennemann (vgl. (10a)). Insbesondere soll die Behauptung belegt werden, dass /i/ - phonetisch realisiert als $[\mathrm{j}]$ - weiterhin im Ansatz erscheint, und nur der Reflex von historischem / $\mathrm{u} /$ in Ansatzposition als Frikativ reanalysiert worden ist (vgl. (10b)):

$$
\begin{aligned}
& \text { a. Vennemann (1988, S. 16): } \\
& \text { /iār / > /jar/ Jahr } \\
& \text { /unal/ > / val/Wall }
\end{aligned}
$$

b. Hier vertretene Sicht: $/ \mathrm{i} / \bar{a} \mathrm{r}[\mathrm{j}] \bar{a} r>/ \mathrm{i} / \mathrm{ahr}[\mathrm{j}] \mathrm{ahr}$ $/ \mathrm{u} / \mathrm{al}[\mathrm{w}] \mathrm{al}>/ \mathrm{v} / \mathrm{al}[\mathrm{v}] \mathrm{all}$

Die hier angenommene Regel für die Kodasilbifizierung hoher Vokale im Deutschen ist in (11) dargestellt. Sie geht mit der Analyse der so genannten schließenden Diphthonge als biphonemische Sequenzen /ai/, / au/ und / oi/ einher:

$$
\begin{array}{llr}
\text { mögliche Koda } & & \text { notwendiger Nukleus } \\
\text { i }>\text { u }>\text { y }>\text { I, }, \text {, Y }
\end{array}
$$

Die vorliegende Studie ist wie folgt strukturiert. In den Abschnitten 2 und 3 sollen die in (9b) bzw. (11) skizzierten Grammatiken für das Deutsche motiviert werden. Der Gegenstand von Abschnitt 4 ist die Herleitung der entsprechenden Allophonie als Funktion von unterschiedlichen prosodischen Positionen. In Abschnitt 5 geht es um potenzielle Gegenbeispiele und lexikalische Ausnahmen. Eine kurze Zusammenfassung der Ergebnisse erfolgt in Abschnitt 6.

\section{Zur phonemischen Zuordnung von [j] im Deutschen}

In diesem Abschnitt soll zunächst die in der deutschen linguistischen Literatur übliche Analyse des initialen Lauts in Wörtern wie ja als Frikativ infrage gestellt werden. In Abschnitt 2.2 wird die phonologische Evidenz für eine Analyse dieses Lauts als /i/-Allophon präsentiert. In Abschnitt 2.3 erfolgt ein kurzer Vergleich mit dem labiodentalen Frikativ [v].

\subsection{Zur Klassifizierung von $[\mathrm{j}]$ als Frikativ}

Vergleicht man die Umschriften der deutschen Wörter Yak, Yucca und Yoga mit den Entsprechungen in französischen und englischen Aussprachewörterbüchern, so zeigt sich, dass das wortinitiale Segment in letzteren ausnahmslos als Approximant (bzw. „Halbvokal“ oder „Halbkonsonant"), im Deutschen hingegen stets als Frikativ klassifiziert wird. ${ }^{8}$

Diese unterschiedliche Klassifizierung des initialen Segments ist partiell dadurch verdeckt, dass das IPA-Symbol [j] bis 1989 sowohl für den palatalen Approximanten (ohne Friktion), 


$\begin{array}{lll}\text { Französisch }^{9} & \text { Englisch }^{10} & \text { Deutsch }^{11} \\ {[\mathrm{j}] \text { ak }} & {[\mathrm{j}] \mathrm{ak}} & {[\mathrm{j}] \mathrm{ak}} \\ {[\mathrm{j}] \text { ucca }} & {[\mathrm{j}] \text { ucca }} & {[\mathrm{j}] \text { ucca }} \\ {[\mathrm{j}] \text { oga }} & {[\mathrm{j}] \text { oga }} & {[\mathrm{j}] \text { oga }}\end{array}$

Der klare Unterschied in der Repräsentation des jeweiligen initialen Segments ist bemerkenswert, finden sich doch besonders in der nichtdeutschen sprachvergleichenden Literatur einige Hinweise auf die phonetische Ähnlichkeit der fraglichen Laute. Moulton (1962, S. 14) äußert sich wie folgt:

[...] [j] is the obvious symbol for the initial sound of German jetrt, ja, jung, etc.; and since English [j] and German [j] are to all intents and purposes phonetically identi$\mathrm{cal}$, it would be undesirable to symbolize them differently.

Auch Fouché (1956, S. XXX) charakterisiert die in (12) veranschaulichten wortinitialen Segmente im Französischen, Englischen und Deutschen als „plus ou moins approximatifs“. Ebenso Delattre (1965, S. 81), in seiner sprachvergleichenden phonetischen Studie, klassifiziert die fraglichen Laute auch im Deutschen als nichtfrikativische, ungerundete, palatale Sonoranten.

Neuere phonetische Studien bestätigen, dass die Klassifikation des so genannten ,deutschen $/ \mathrm{j} /$ “ als Obstruent zumindest in der heutigen Standardsprache nicht berechtigt ist (siehe Abschnitt 4). So klassifizieren Kohler (1995, S. 155), ${ }^{12}$ Pompino-Marschall (1995, S. 189) und Mücke (1998) das prävokalische $[\mathrm{j}]$ im Deutschen als palatalen Approximanten. Woher aber kommt die Vorstellung, dass es sich um einen Frikativ handle? In den einschlägigen Belegen findet sich schon von Beginn an der Bezug auf den stimmlosen palatalen Frikativ [c]]:

Man kann das $j$ auch so betrachten, als wenn es ein bloßes $c h$ wäre, bey dem die Stimme mitlautet. (von Kempelen 1791, S. 209)

Bei Siebs (1905, S. 66) erlangt dieser Bezug einen normativen Charakter:

Der dem stimmlosen $c h$ entsprechende stimmhafte palatale Reibelaut ist $j$.

Seither ist die analoge Behandlung der Lautpaare [f]-[v], [s]-[z], [J]-[3] und $[\mathrm{c}]-[\mathrm{j}]$ in den Beschreibungen des deutschen Lautsystems gang und gäbe

als auch für den palatalen Frikativ verwendet wurde. Erst dann wurde für letzteren Laut ein eigenes Symbol [j] eingeführt, eine Neuerung, die allerdings in den Arbeiten zum Deutschen seither kaum beachtet worden ist. Der Gebrauch der Symbole [j] und [j] in der Tabelle in (12) entspricht somit nicht unbedingt dem tatsächlichen Gebrauch der Symbole in den fraglichen Aussprachewörterbüchern, sondern repräsentiert die jeweiligen intendierten Laute (Approximant versus Frikativ).

$9 \quad$ Lerond (1980); Warnant (1987).

10 Kenyon/Knott (1953); Wells (2000).

11 Viëtor (1921); Siebs (1957); Mangold (2005); Muthmann (2006); Krech et al. (2009).

12 Die von ihm zuvor vertretene Analyse von [j] als Frikativ (1977, S. 159) hat Kohler somit revidiert. 
(vgl. Viëtor 1921, S. XVII; von Essen 1962; Vennemann 1982, S. 274; Wiese 1996, S. 23; ${ }^{13}$ Bußmann (Hg.) 2002, S. 370; Maas 1999, S. 192). Von dort scheint es nur ein kurzer Schritt zur Phonemanalyse in (13), wo der fragliche Palatal als stimmhafter Frikativ beschrieben wird, der in Opposition zum stimmlosen palatalen Frikativ steht (vgl. Philipp 1974, S. 36; Meinhold/ Stock 1982, S. 120 ff.; Ramers/Vater 1995, S. 74+14).

$\begin{array}{lcccc} & \text { labiodental } & \text { alveolar } & \text { alveopalatal } & \text { palatal } \\ \text { stimmlos } & / \mathrm{f} / & / \mathrm{s} / & / \mathrm{S} / & / \mathrm{c}, \mathrm{x} / \\ \text { stimmhaft } & / \mathrm{v} / & / \mathrm{z} / & / \mathrm{3} / & / \mathrm{j} /\end{array}$

Die Phonemanalyse in (13) ist in allen neueren Nachschlagewerken zum Deutschen, einschließlich der Aussprachewörterbücher (vgl. Muthmann 1996, S. 6; Mangold 2005, S. 43 ff.; Krech et al. 2009, S. 29) und der Grammatiken (vgl. Zifonun/Hoffmann/Strecker 1997, S. 172 ff.; Duden-Grammatik 2009, S. 34) kommentarlos übernommen. Diese Entscheidungen muten seltsam an, scheint die Phonemanalyse in (13) doch in erster Linie durch die Symmetrie des Systems begründet, ein schwaches Argument, das bei näherem Hinsehen sogar eher gegen die Analyse spricht. So ist die Stimmhaftigkeitsopposition für Frikative im Deutschen im Vergleich zu Plosiven ohnehin schwach ausgelastet, ${ }^{15}$ wobei diese Auslastung umso schwächer wird, je weiter hinten die Artikulationsstelle ist. Für Glottale ist die Opposition nicht belegt; für Alveopalatale ist sie auf nur wenige, oft unstabile Lehnwörter beschränkt. Selbst bei alveolaren Frikativen ist die funktionale Belastung der Opposition sehr gering, ist sie doch in wortinitialer Position neutralisiert. Das Fehlen der Opposition bei den palatalen Frikativen wäre somit eher charakteristisch für das Deutsche. ${ }^{16}$

13 Wiese (1996) klassifiziert einerseits, im Einklang mit der gängigen Auffassung, den Laut [j] als stimmhaftes Gegenstück zu [ç], analog zu Paaren wie [v]-[f], [z]-[s] oder [3]-[S] (siehe 1996, S. 23). Andererseits führt er, in Anlehnung an Trubetzkoy (1958 [1938], S. 64), [i] auch als Allophon von /i/ auf (Wiese 1996, S. 18), schließlich, in Anlehnung an Hall (1992), betrachtet er [j] noch als Allophon des phonetisch kurzen Vokals wie in Mitte (1996, S. 235). Die Vereinbarkeit dieser Klassifizierungen ist unklar.

14 In der Phonemtabelle (1995, S. 81) klassifizieren die Autoren das / j/ als stimmhaftes Oppositionsglied von / $\mathrm{k} /$, vermutlich ein Tippfehler.

15 Auch im Englischen ist die Stimmhaftigkeitsopposition für Frikative, nicht aber für Plosive,

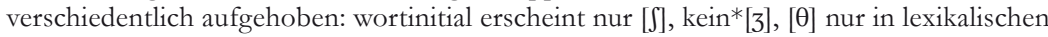
Wörtern, [ð] nur in Funktionswörtern.

16 Dass der Vergleich der Paare in (13) hinkt, lässt sich bereits an Ungereimtheiten in den Beschreibungen ablesen. So beschreibt Philipp (1974, S. 42) die Distinktivität bei labiodentalen Frikativen wie folgt: ,/f/ ist stimmlos, /v/ hingegen stimmhaft.“ Bei den palatalen aber schreibt sie: , /j/ ist stimmhaft, [ç] ist stimmlos.“ Hier werden zunächst zwei Phoneme, dann aber ein Phonem mit einem physikalischen Sprachlaut verglichen. 


\title{
2.2 Das Alternationsverhalten und die Distribution von [j]
}

Zieht man die gesamte phonologische Evidenz in Betracht, so ergeben sich klare Unterschiede in der Relation zwischen den paarweise angeordneten Frikativen in den ersten drei Spalten in (13) und dem Paar [c] -[j]. So alternieren stimmhafte Frikative aufgrund der so genannten Auslautverhärtung mit stimmlosen Frikativen wie in (14a), [j] hingegen alterniert nie mit einem stimmlosen Frikativ. ${ }^{17}$

a. archi[.v]ieren archivieren

hau[.z]ieren hausieren

enga[.3]ieren engagieren

\author{
Archi[f.] Archiv \\ $\mathrm{Hau}[\mathrm{s}$.$] Haus$ \\ Enga[s.]ment Engagement \\ Detá[i] Detail \\ Emá[i] Email
}

b. deta[.j]íeren detaillieren ema[.j]íeren emaillieren

In den wenigen Fällen, wo überhaupt eine Alternation feststellbar ist, alterniert [j] mit /i/-Allophonen. In (14b) alterniert [j] in der Ansatzposition nach offenem Vokal mit wortfinalem unsilbischem [i]. Aufschlussreich ist auch die Adaption der Lehnwörter in (15) aus dem Hebräischen, wo [j] nach gespanntem Vokal mit silbischem /i/ alterniert. Die für tatsächliche Frikative zu erwartende Alternation ist in (15b) veranschaulicht:
a. Go[.j] ím Gojim
Gó[.i.] Goi
b. Moscha[.v]ím Moschawim
Móscha[f.] Moschaw

Das vor vokalisch anlautenden Suffixen beobachtbare Alternationsverhalten in (14) und (15) wird durch die Bildung der Stammformen vor konsonantisch anlautenden Suffixen in (16) bestätigt. Letztere Suffixe erscheinen in Kombination mit bestimmten Stammformen, die sich aus den entsprechenden freistehenden Wortformen abstrahieren lassen und eigene Silbifizierungsdomänen bilden. Derlei Abstraktionen beinhalten notwendig die Tilgung des finalen Schwa, wodurch reguläre Stimmhaftigkeitsalternationen bedingt sind (vgl. (16a)). Die Beobachtung, dass *Kö[ç.]lein, *Medäl[ç.]lein, im Gegensatz zu den entsprechenden Bildungen in (16a) nicht nur ungebräuch-

$17 \quad$ Die Phoneme /f/ $/$ /v/, /s/-/z/, / / / /3/ alternieren zudem regelmäßig in Flexionsparadigmen (vgl. doo[.v]er doofer - doo[f.] doof, Ner[.v]en Nerven - Ner[f.] Nerv, bei[.3]er beiger - bei[f.] beige). Hier ist kein Vergleich möglich, da [j] vor Flexionsendungen nicht vorkommt. Werden prävokalisches [j] und postvokalisches [i] als /i/-Allophone in Ansatz- bzw. Kodaposition analysiert, ist diese Lücke leicht erklärt: Nach Vokal wird stammfinales /i/ vor silbischen Flexionsaffixen stets als Koda silbifiziert (vgl. [nəi. .ə] neue, [taun.ə] Taue). Nach Konsonant wird stammfinales /i/ stets als Nukleus silbifiziert, was bei Adjektiven dazu führt, dass keine flektierten Formen existieren (vgl. uni, mini). Die weitere Generalisierung ist hier, dass alle auf Vollvokal auslautenden Adjektive indeklinabel sind (vgl. prima, rosa, [oké:] ,okay). Die relevanten Substantivflexionsparadigmen enthalten nur das unsilbische Suffix /s/ (Gummis, Okapis). 
lich, sondern unakzeptabel sind, spricht gegen die Annahme einer Opposition $/ \mathrm{j} /-/$ ç/ im Deutschen. Bestünde eine solche Opposition, gäbe es keinen erkennbaren Grund, die Diminutivformen in (16b) zu vermeiden.
a. Sto[.v]e Stove
Do[.z]e Dose
Stö[f.]lein Stö̈lein
Gara[.3]e Garage
Dö[s.]lein Döslein
Garä[S.]lein Garägelein
b. Ko[.j]e Koje
Meda[1.j]e Medaille

Die Beobachtung, dass die Stammbildung in (16b) insgesamt blockiert scheint, deutet auf ein phonologisches Problem hin, das bei stammfinalen Frikativen in (16a) nicht besteht. Unter der Annahme, dass [j] als /i/-Allophon zu analysieren ist, ließe sich das Problem als ein die Silbifizierung betreffendes Dilemma erklären: In der finalen Position der Silbifizierungsdomäne kann /i/ nicht als Ansatz erscheinen (*Kö[.j]lein), nach [ø] oder Konsonant scheidet auch die Kodaposition aus (*Kö[i.].]lein, Medäl[i.].lein). Dass /i/ auch nicht als Nukleus silbifizierbar ist, weist auf eine Beschränkung bei der Abstraktion von Stammformen hin: Offensichtlich darf diese nicht mit einer Erhöhung der Silbenzahl gegenüber der prosodischen Organisation der fraglichen Segmente in der Basis einhergehen (*Kö[.i.]lein, $\left({ }^{*}\right.$ Medäl[i.]lein $){ }^{18}$

Dass [j] im Deutschen als Allophon des Phonems /i/ fungiert, wird vor allem durch die komplementäre Verteilung mit potenziell silbischen /i/-Allophonen deutlich. So erscheint in den oben aufgeführten deutschen Aussprachewörterbüchern in übereinstimmender Weise wortintern das Symbol [j] bzw. [j] nur nach gespanntem Vokal oder den Sonoranten /1/ und /n/, sofern diesen ein ungespannter Vokal vorangeht; sonst erscheint unsilbisches [i] oder silbisches [i] in prävokalischer Position. Hier bietet sich die in (17) veranschaulichte prosodische Analyse an: Die Vermeidung der tautosilbischen Folgen /li/ oder /ni/ wird durch die Silbifizierung der Sonoranten in Kodaposition erreicht. Diese Option ist nach gespanntem Vokal wie in (17b) nicht gegeben, da solche Vokale im Deutschen auf der phonemischen Ebene nur in offenen Silben auftreten. Folglich erscheinen /1/ oder / $\mathrm{n} /$ in Ansatzposition vor /i/, das zusammen mit dem vorangehenden Konsonanten einen komplexen Ansatz bildet (vgl. Abschnitt 4.2). Eine solche Struktur ist auch für alle übrigen Fälle anzunehmen, in denen prävokalisches /i/ nach postvokalischen Konsonanten auftritt (vgl. (17c)).

\footnotetext{
18 Dass im Deutschen auf Stammbildung infolge unlösbarer Probleme bei der prosodischen Organisation der Segmente schlicht verzichtet wird, ist durch weitere Lücken in der Stammbildung bestätigt (siehe Wi/t.v/e Witwe, aber *Wi/tf./lein, Dra/x.m/e Drachme, aber *Dra/xm./lein).
} 
(17)

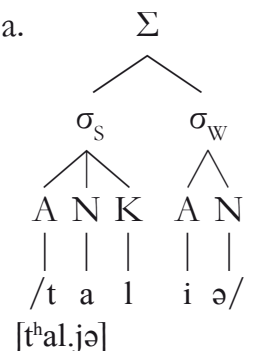

Taille b.

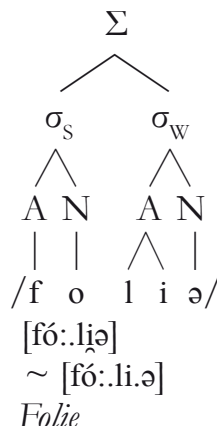

c.

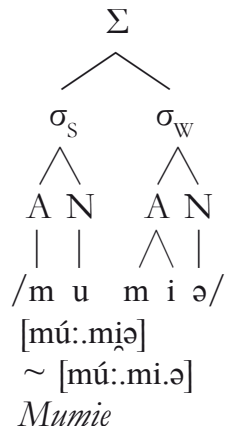

Im Folgenden bezeichnet das Symbol [j] einen Approximanten, der als phonetische Realisierung des Phonems / i/ am Silbenanfang auftritt. In anderen Kontexten, insbesondere im wortinternen komplexen Ansatz oder in der Koda, wird die Realisierung des Phonems /i/ auf der phonetischen Ebene mit dem Symbol [i] repräsentiert.

Phonologisch äußern sich die relevanten Silbifizierungsbeschränkungen darin, dass gespannte und ungespannte Vokale vor Konsonant-/i/Sequenzen nur dann potenziell kontrastieren, wenn die Silbe betont und der Konsonant / $/$ oder /n/ ist (vgl. (17a/b)). Weitere Beispiele sind in (18) aufgeführt. Vor allen anderen Konsonant-/i/-Sequenzen ist der Gespanntheitskontrast aufgehoben: nur gespannte Vokale erscheinen (vgl. $(17 \mathrm{c}))$.

$\begin{array}{ll}\text { a. Em[ál.j]e } & \text { Emaille } \\ \text { Bred[úl.j]e } & \text { Bredonille } \\ \text { Van[íl.j]e } & \text { Vanille } \\ \text { K[ón.j]ak } & \text { Kognak } \\ \text { B[án.j]o } & \text { Banjo }\end{array}$

\begin{tabular}{|c|c|}
\hline D[á:.li]e & Dablie \\
\hline Magn[ó:.li] & Magnoli \\
\hline Fam[í:.li]e & Familie \\
\hline Col[ó:.ni]a & Colonia \\
\hline Ger[á:.ni]]e & $G e$ \\
\hline
\end{tabular}

Angesichts der Beispiele in (18) mag man einwenden, dass es sich hier lediglich um fremdsprachlich, bzw. schriftsprachlich begründete Muster handle. Dagegen spricht aber der Umstand, dass selbst in den wenigen Fällen von Variation ein ungespannter Vokal stets mit unsilbischem [j] und ein gespannter Vokal stets mit potenziell silbischem Vokal korreliert (z.B. Stamp[íl.j]e versus Stamp[í:li]e Stampiglie). Auch der Sprachvergleich bestätigt die Annahme, dass es sich um eine kontextuell bedingte Verteilung handelt. Die englischen Daten in (19) veranschaulichen die gleiche Verteilung: Die einzigen Konsonanten, die vor notwendig unsilbischem [j] vorkommen, sind [n] und [1], und zwar nur wenn diesen ein ungespannter Vokal vorangeht wie in (19a). ${ }^{19}$ Der

19 Notwendig unsilbisches [j] in [féiljər] failure ,Versagen` oder [bihéivjər] behavior ,Benehmen` ist keine Ausnahme, da dieses [j] den Ansatz eines Suffixes bildet und deswegen außerhalb des phonologischen Wortes des Stammes erscheint (d.h. (feil) $)_{\omega} /$ irr/, bi(heiv) $\omega_{\omega} /$ irr/). 
Verdacht, dass die Zusammenhänge durch die Orthographie gesteuert sein könnten, ergibt sich im Englischen nicht: In (19) wird sowohl notwendig unsilbisches [j] als auch potenziell silbisches [i] durch das gleiche Graphem $<\mathrm{i}>$ symbolisiert: ${ }^{20}$
a. [ún.jən $] *[\Lambda ́ n i . ə n]$
b. [mí:.nirəl] $\sim$ [mí:.ni.əl]
menial niedrig
onion Zwiebel
[stál.jən] *[stǽli.ən]
stallion Hengst
[éi.liñon] [éi.li.ən]
alien fremd

Geht kein ungespannter Vokal voran wie in (19b) oder erscheint statt /n/ oder /1/ ein anderer Konsonant, kann der palatale Gleitlaut im Englischen immer silbisch ausgesprochen werden (vgl. triv[i] al trivial, cam[i] on camion). Die englischen Daten bestätigen somit eine besondere Beschränkung gegen die Silbifizierung der Sonoranten /1/ oder /n/ im Ansatz vor /i/. Verletzt wird diese Beschränkung nur, wenn die Kodaposition aufgrund der vorangehenden segmentalen Struktur nicht verfügbar ist, sei es, weil ein gespannter Vokal vorangeht, oder sei es, weil die Koda bereits durch einen Konsonanten besetzt ist, wie in (20). Auch in (20) kann /i/ nach /n/ immer silbisch ausgesprochen werden, im Gegensatz zu den Fällen in (18a):

$$
\begin{array}{ll}
\mathrm{He}[\mathrm{R} . n \mathrm{n}] \mathrm{e} & \text { Hernie } \\
\text { Vi[1.ni]us } & \text { Vilnius }
\end{array}
$$

Eine allophonische Verteilung zwischen notwendig unsilbischem [j] und potenziell silbischem [i] ist somit auch für das Deutsche anzunehmen. Das letztere Allophon ist durch den segmentalen Kontext weniger eingeschränkt und kommt entsprechend wortintern häufiger vor: Folglich alterniert es auch öfter mit silbischem [i] wie in (21). Dass eine solche Alternation auch für [j] möglich ist, zeigt das Beispiel in (15a).

$$
\begin{aligned}
& \text { a. kolo[.ni]ál kolonial } \\
& \text { melo[.di] ös melodiös } \\
& \text { Ma[.gi]er Magier } \\
& \text { katego[.ri] al kategorial }
\end{aligned}
$$
b. Kolon[í:] Kolonie
Melod[í:] Melodie
Mag[í:] Magie
Kategor[í:] Kategorie

Alternationen zwischen unsilbischem [i] das als Zweitelement eines komplexen Ansatzes erscheint, und silbischem [i] finden sich besonders häufig für Substantive und deren Ableitungen vor dem Suffix -ier wie in (22a/b).

$20 \quad$ Die Umschriften in (19) sind mit Wells (2000) kompatibel. Anzumerken ist lediglich, dass im Britischen silbisches /i/ in einigen Wörtern des Typs in (19a) als Variante zugelassen ist. 
(22)

a.

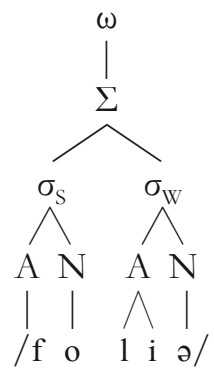

[fó::lìn]

Folie b.

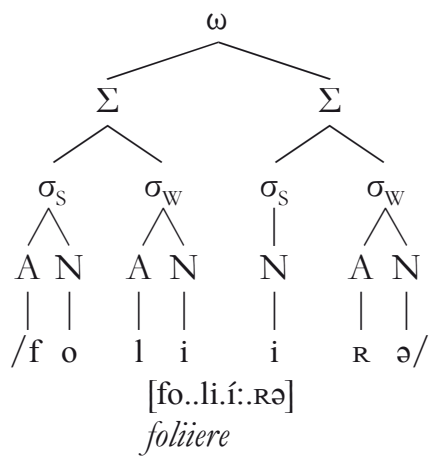

Weitere Beispiele sind in (23) gegeben:

\begin{tabular}{|c|c|}
\hline & \\
\hline . Pla[.gi]át & Plagiat \\
\hline $\begin{array}{l}\text { Va[.ri]án } \\
\text { ini[-.tsilál }\end{array}$ & $\begin{array}{l}\text { Varianz } \\
\text { initial }\end{array}$ \\
\hline Lí[.ni]e & Linie \\
\hline Prấ[.mi]e & Prämie \\
\hline
\end{tabular}

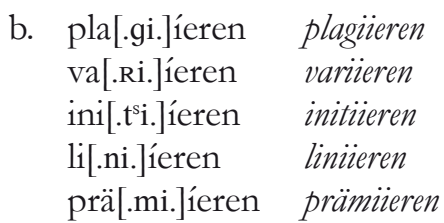

Die regelmäßige Alternation zwischen unsilbischem [i] und silbischem [i] bestätigt die Analyse beider Laute als Allophone des Phonems /i/. Die Nichtexistenz entsprechender [j]-[i]-Alternationen vor -ier ist ausschließlich durch den unterschiedlichen segmentalen Kontext bedingt. Nach /1/ oder $/ \mathrm{n} /$, sofern diesen Sonoranten ein ungespannter Vokal vorangeht, kommen Alternationen wie in (23) nicht vor: Stattdessen erscheint nur unsilbisches [j] (siehe $(24 \mathrm{a} / \mathrm{b}){ }^{21}$ Auch zwischen Vollvokalen wie in der Variante in (24c) erscheint nur unsilbisches [j]:

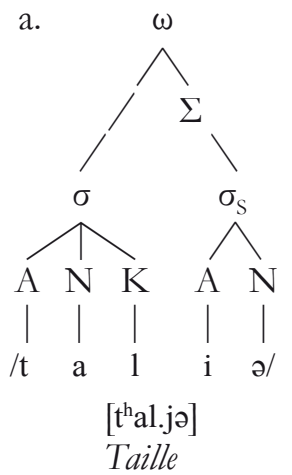

b.

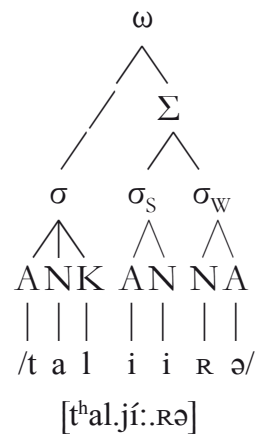

tailliere c.

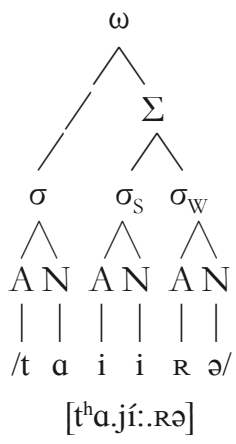

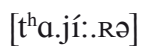

$21 \quad$ Das Wort all[i.] ieren alliieren ist eine Ausnahme. 
Der Grund für die fehlenden Alternationen mit silbischem [i] in (24a/b) liegt in der durch die Phonemfolgen bedingten Struktur des Silbenansatzes: In (24a) enthält dieser nur /i/ während das /i/ in (22a) in Zweitposition eines komplexen Ansatzes erscheint. Weitere Beispiele für nichtalternierende Vokale, die alleine im Ansatz erscheinen, sind in (25) gegeben:
a. Emá[1.j]e Emaille
Patróu[1.j] Patrouille
Medá[1.j]e Medaille
bri[1.j]ánt brillant
b. ema[1.j] íeren ${ }^{22}$ emaillieren patrou[1.j]1íeren patroullieren meda[1.j]íeren medaillieren bri[1.j]1íeren brillieren

Die Beschränkung, die der unterschiedlichen Silbifizierung des prävokalischen /i/ in tailliere versus foliiere letztlich zugrunde liegt, betrifft die silbische Organisation aufeinanderfolgender identischer Vokale. Im Deutschen wird /i/ vor /i/ regulär als einfacher Ansatz silbifiziert wie in (26a). Ergäbe sich dadurch aber ein komplexer Ansatz wie in (26b), so wird /i/ stattdessen als Nukleus silbifiziert, so dass ein /i.i/-Hiatus entsteht (vgl. (26c)). Diese Beschränkung gilt nicht vor anderen silbischen Vokalen, wo vorangehendes /i/ auch dann als Ansatz silbifiziert wird, wenn dieser komplex ist (vgl. (26d)).

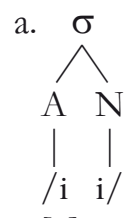

[ji]

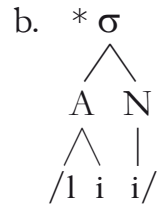

$*[$ lii $]$

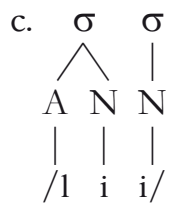

[li.i $]$

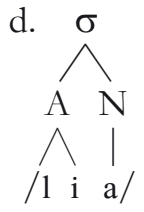

[lia]

Beispiele für die Silbenstrukturen in $(26 \mathrm{a} / \mathrm{c} / \mathrm{d})$ sind in $(27 \mathrm{a} / \mathrm{b} / \mathrm{c})$ gegeben. Im Gegensatz zu früheren Analysen des Unterschieds in (27a) versus (27b) gibt es in den hier vorgestellten Generalisierungen keinerlei Bezug auf morphologische Struktur. ${ }^{23}$ Stattdessen sind die Silbenstrukturen ausschließlich durch den segmentalen Kontext innerhalb der jeweiligen Silbifizierungsdomänen determiniert:

(27) a. deta[.jí:]re detailliere, e[.ji ] ziere ejiziere, Feda[.jí:] Fedajin, Go[.ji: $] m$ Gojim

b. fo[.li.í:]re foliiere, [ji.í:]t Schiit, Ko[.ni.í:]n Konïn, [li.í:] re liiere

c. Fo[.lìn] Folie, Ra[.dio] Radio, Sa凡.mia]k Salmiak

22 Für das Wort Emaille gibt es zwei lexikalische Varianten, [emáljə] und [emái]. Entsprechend unterscheiden sich sämtliche Ableitungen (z.B. [emaljí:Rən] [emají:Rən] emaillieren, [emáljǿø] [emajǿø Emailleur).

23 Vgl. Hall (2005), der phonetische [ji]-Sequenzen mit „nichtderivierten“ Strukturen in Verbindung bringt. 
Eine analoge Beschränkung lässt sich im Englischen feststellen, wo /u/ in allen Silbenpositionen erscheinen kann. In (28a) ist die reguläre Ansatzsilbifizierung von $/ \mathrm{u} /$ vor $/ \mathrm{u} /$ dargestellt. In (28b) wird gezeigt, dass ein solcher Ansatz nicht komplex sein darf, trotz des regulären Vorkommens komplexer Ansätze, wenn nichtidentische Phonemsequenzen betroffen sind, wie in $(28 \mathrm{c}):^{24}$
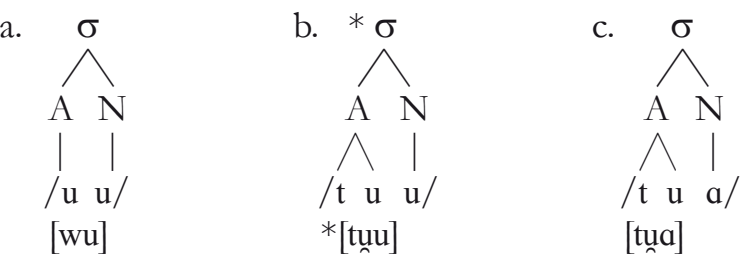

Beispiele für die Silbifizierung in (28a) sind in (29a) gegeben. In (29b) wird gezeigt, wie das Problem, das die prosodische Organisation von Segmentfolgen wie in (28a) aufwirft, im Englischen gelöst wird: Der erste der beiden identischen Vokale wird nicht, wie im Deutschen, als Nukleus silbifiziert (vgl. (26c)), sondern schlicht getilgt:

$$
\begin{aligned}
& \text { a. [wu:] woo, [wu:]nd wound, [wú:]zy woosy } \\
& \text { b. *[tuu:] } \Rightarrow \text { [tu:] two, aber: [tur]n twin, [tuc]lve twelve, } \\
& \text { [tuc]nty twenty, [tuai]ce twice } \\
& * \text { [huu:] } \Rightarrow \text { [hu:] who, aber: [hự]n when, [hun]t what } \\
& \text { [hur]ch which, [huse]re where }
\end{aligned}
$$

Die in (29b) veranschaulichte Strategie zur Vermeidung von komplexen Ansätzen vor identischen Vokalen ist gelegentlich auch im Deutschen zu beobachten (vgl. prä[.mí:.] ren, neben prä[.mi.í:.] ren).

Weitere Belege für die komplementäre Verteilung silbischer und unsilbischer /i/-Allophone sind in Tabelle (30) aufgeführt. Geht nur ein Konsonant, bzw. eine Verbindung mit fallender Sonorität voran, wie in (30a), so wird das /i/ als Teil eines komplexen Ansatzes organisiert und daher unsilbisch gesprochen. Geht aber eine Verbindung mit (stark) steigender Sonorität voran, wie in (30b), so bildet diese Verbindung bereits einen komplexen Silbenansatz. Da Ansätze höchstens zwei Phoneme enthalten können, gibt es für das /i/ dann gewissermaßen keinen Platz mehr. Folglich wird es als Nukleus organisiert und silbisch gesprochen: ${ }^{25}$
a. En[.tsi]an
Enzian
$\mathrm{O}[\cdot \mathrm{pi}] \mathrm{um}$
Opium
b. Schen[.dRi.]an Pro[.pri.]um Schlendrian
Proprium

24 Das Vorkommen von [suu]-Folgen wie in swoop, swoon, swoosh deckt sich mit weiterer Evidenz, die zeigt, dass /s/ nicht Teil der Silbe ist, sondern einen so genannten Appendix bildet.

25 Die in (30) veranschaulichten Unterschiede hinsichtlich der Silbischkeit sind in allen deutschen Ausprachewörterbüchern markiert. 
Die gleiche Beschränkung hinsichtlich der maximalen Anzahl von Phonemen im Silbenansatz gilt auch im Französischen: Nach einem silbeninitialen Konsonanten ist das /i/ unsilbisch (vgl. (31a)), nach zweien ist es silbisch (vgl. (31b)): ${ }^{26}$
a. hui[.ti]ème
buitième
achtens
b. qua[.tri.]ème
quatrième viertens

Im Gegensatz zum Deutschen findet sich dieser Effekt im Französischen auch in wortinitialer Position (siehe (32)). Zudem ist er dort nicht auf das /i/ beschränkt, sondern betrifft auch /u/ und /y/ (Fouché 1956, S. 4; Tranel 1987, S. 116):
a. $[$ lie $] / *[$ li.e $]$ lier
,binden
[lue $] / *[$ lu.e $]$ lover
,mieten
[Rye]/*[Ry.e] ruer
,treten
b. $[$ pli.e $] / *[$ plie $] \quad$ plier
,falten
[klu.e $/ *[$ klue $]$ cloner
,nageln
[gry.o]/*[gryo] gruau
,Grütze

Die komplementäre Verteilung von notwendig silbischem [i], potenziell silbischem [i] und notwendig unsilbischem [j] im Deutschen spricht somit gegen die Klassifizierung von [j] als eigenständiges Phonem, das als stimmhaftes Glied der Opposition $/ \mathrm{j} /-/$ ç/ fungiert. Stattdessen repräsentiert $[\mathrm{j}]$, in Übereinstimmung mit der Darstellung in (9), nach wie vor das Phonem /i/ im Silbenansatz.

\subsection{Neutralisationserscheinungen im Vergleich mit [v]}

Die Analyse des Standarddeutschen in (9) ist durch die Annahme eines Sonoritätsunterschieds zwischen /i/ und / $\mathrm{u} /$ motiviert: Während /i/ weiterhin im Ansatz erscheinen kann, ist eine solche Silbifizierung für das sonorere /u/ nicht mehr zulässig, wobei die historischen Reflexe von /u/-Allophonen in Wörtern wie Wall als konsonantische /v/-Allophone reanalysiert worden sind. Phonetisch ist der Unterschied dadurch begründet, dass $[\mathrm{j}]$ in Jabr ein Approximant, [v] in Wall hingegen ein Frikativ ist (Pompino-Marschall 1995, S. 189). In phonologischer Hinsicht wurde der Unterschied bereits dadurch motiviert, dass [v] und [f] regelmäßig alternieren, [j] jedoch nie mit stimmlosen Frikativen alterniert (vgl. (14-16)). Hinsichtlich der Phonotaktik zeigt sich, dass / $/$ vor / R / mit anderen Frikativen kontrastiert (siehe (33a)), diese Position für [j] aber kategorisch ausgeschlossen ist:

\footnotetext{
26 In den französischen Aussprachewörterbüchern werden unsilbische Vokale durchweg mit den Symbolen [j], [w] und [ $\mathrm{\Psi}]$ repräsentiert. Ich folge hier der Konvention, diese Symbole nur für Vokale zu verwenden, die alleine im Ansatz erscheinen.
} 
a. [vR] ack Wrack, [vR]ingen wringen $[\mathrm{fR}]$ ack Frack, [fR] isch frisch

b. $*[j R]$ [çR] isam Chrisam, [çR]ysoberyll Chrysoberyll

Während das Auftreten von [v] vor Konsonant in (33a) die Annahme einer historischen Reanalyse als Frikativ stützt, so stellen die Ansatzverbindungen in (34a) eine solche Klassifikation eher in Frage. Zwar finden sich PlosivFrikativ-Folgen auch in Lehnwörtern (siehe (34b)), aber tautosilbische Fortis-Lenis-Verbindungen kommen sonst im Deutschen nicht vor. ${ }^{27}$
a. [kv]al Qual
[Jy]an Schwan
$\left[\mathrm{t}^{\mathrm{s}} \mathrm{v}\right]$ ar zwar
[tv]ist Twist

b. [ps]alm Psalm

[ks]anthippe Xanthippe

Dennoch zeigt sich, dass das Zweitglied in deutsch Twist, Schwa oder Quark im Gegensatz zu englisch twist, shwa, quark als Frikativ gesprochen wird. ${ }^{28}$ Auch die Neutralisationserscheinungen in (35) weisen darauf hin, dass sich postkonsonantisches [v] wie ein Obstruent verhält. So folgt die Verbindung [RV] immer auf einen ungespannten Vokal (siehe (35a)); intervokalischen Verbindungen [Ri] hingegen geht gewöhnlich ein gespannter Vokal voran (siehe (35b)): $:^{29}$
a. K[úr.v]e Kurve

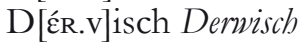
N[ér.v]en Nerven
S[ER.v]iétte Serviette

b. F[ú:.Ri]e Furie

Z[é:.Ri]um Zerium

F[é:.ri]en Ferien

$\mathrm{s}[\mathrm{e} . \mathrm{Ri}] \mathrm{ös}$ serï̈s

Die ungespannten Vokale in (35a) lassen auf eine geschlossene Silbe schlieBen, während die gespannten Vokale in (35b) eine offene Silbe indizieren. Daraus folgt, dass die intervokalische Folge [Rv] heterosilbisch, [Ri] hingegen als komplexer Ansatz silbifiziert wird, in Übereinstimmung mit den Analysen von [v] als /v/-Allophon und [i] als /i/-Allophon. Diese Interpretation wird durch den Effekt einer Permutierung der fraglichen Segmentfolgen bestätigt. Vor / vR/ ercheinen nur gespannte Vokale, was auf eine Silbifizierung der Folge im Ansatz hindeutet; vor /iR/ erscheinen nur ungespannte Vokale, was auf eine heterosilbische Organisation der Folge hindeutet.

$$
\begin{aligned}
& \text { a. L[i.vR]ée Livre } \\
& \text { [ø̛.:vR]e CEvre } \\
& \text { L[ú:.vR]e Lowvre }
\end{aligned}
$$

$$
\begin{array}{ll}
\text { b. } & \text { [ói.r]o Euro } \\
& \text { K[ái.r]o Kairo } \\
& \text { K[áu..R]i Kauri }
\end{array}
$$

\footnotetext{
${ }_{27}$ Die partielle Stimmlosigkeit ist assimilatorisch und betrifft nur die phonetische Ebene.

28 Bei der Aussprache von deutsch Quark berühren die oberen Schneidezähne die Unterlippe. Bei der Aussprache von englisch quark ist das nicht der Fall.

29 Terrier ist eine lexikalische Ausnahme, es sei denn, /i/ ist silbisch vor dem Suffix -er (siehe Krech et al. 2009). Der Status von Karriere und Barriere ist schwer zu beurteilen, da der Gespanntheitskontrast bei offenen Vokalen qualitativ kaum nachweisbar ist und daher vor allem durch die Dehnbarkeit in betonten Silben zu erkennen ist.
} 
Die charakteristischen Unterschiede in der silbischen Organisation der relevanten Phonemfolgen sind in (37) dargestellt: Ob gespannte oder ungespannte Vokale vorangehen, ist eine Folge dieser Organisation.

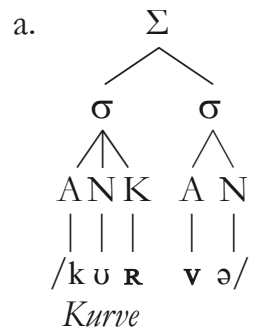

b.

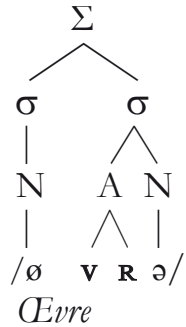

c.

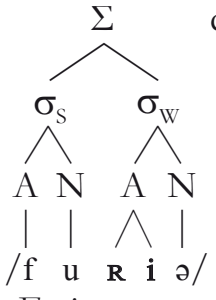

d.

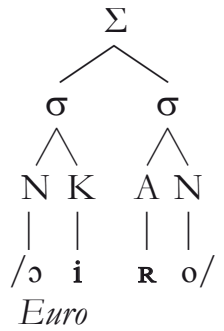

Wie / $/$ / in (35a) erscheinen auch alle übrigen Sonoranten vor / $/$ in der Koda (siehe (38a)). Folglich gehen nur ungespannte Vokale voran.
a. V[úl.v]a Vulva
V[él.v] et Velvet
b. V[ít.v]e Witwe
F[át.v]a Fatwa
Tri[úm.v]ir Triumvir
K[ón.v]oi Konvoi
W[ík.v]am Wigwam
$\mathrm{M}[$ [́k.v]e Mikwe

Auch Obstruenten vor /v/ schließen gewöhnlich die Silbe (siehe (38b)), obwohl Plosive vor Frikativen im Ansatz möglich sind, vgl. (34b). ${ }^{30}$ Nur wenn der Frikativ in der Schrift als Vokal repräsentiert wird, insbesondere <qu> (vgl. R[é:.kv]iem Requiem), erscheint /k/ meist im Ansatz. ${ }^{31}$

Auch in intervokalischer Position unterscheiden sich [v] und [j] hinsichtlich ihrer Kontrastfähigkeit. So kontrastieren [v] und [u] nach kurzem /a/ (vgl. (39a)), während [j] nur nach gespanntem Vokal, [i] hingegen nur nach ungespanntem Vokal erscheint (vgl. (39b)).
a. [káve] Cover - [kháune $]$ Kauer
b. [ko:jə] Koje $-{ }^{*}[\mathrm{ko}: \mathrm{i} \partial]$
[Roiñ Reue - *[Rоjo]

Zusammenfassend kann festgestellt werden, dass [u] und [v] im heutigen Deutschen unterschiedliche Phoneme repräsentieren, [i] und $[j]$ hingegen komplementär verteilt sind. Historisches /u/ in Ansatzposition ist durchgängig als Frikativ reanalysiert worden. Die Beobachtung, dass die auf diese Weise entstandenen markierten Ansatzverbindungen (z.B. > [kv]al Qual) relativ stabil zu sein scheinen, stimmt mit der generellen Toleranz für „,unangepasste“ Ansätze in Lehnwörtern wie Pneumatik, Mnemonik, Khmer, Ptosis oder Namen wie Gwendolin überein.

\footnotetext{
$30 \quad$ Das gilt auch für intervokalische Plosiv-Frikativ-Folgen, wie an den gespannten Vokalen in Wörtern wie K[é:.ps]e Kebse, st[á:.ks]en staksen zu erkennen ist.

31 Auch hier besteht eine Tendenz, die Silbe durch den Plosiv zu schließen (vgl. Koll[j́k.v]ium Kolloquium).
} 


\section{Potenzielle Kontraste zwischen Vokoiden in der Koda}

Im vorangehenden Abschnitt wurde argumentiert, dass /i/ im Deutschen im Ansatz erscheinen kann, / $\mathrm{u}$ / hingegen nicht. Das gesamte Kontrastpotenzial der hohen Vokoide in den Silbenrandpositionen wird in Tabelle (40) verglichen.

$\begin{array}{llc} & \text { Ansatz } & \text { Koda } \\ \text { Deutsch } & / \mathrm{i} / & / \mathrm{i} /-/ \mathrm{u} / \\ \text { Englisch } & / \mathrm{i} /-/ \mathrm{u} / & / \mathrm{i} /-/ \mathrm{u} / \\ \text { Französisch } & / \mathrm{i} /-/ \mathrm{u} /-/ \mathrm{y} / & -\end{array}$

Die Kontraste in (40) sind in (41) veranschaulicht.

\begin{tabular}{|c|c|c|}
\hline Deutsch & $\begin{array}{l}\text { Kontraste im Ansatz } \\
\text { [ja:] }\end{array}$ & $\begin{array}{l}\text { Kontraste in der Koda } \\
\text { [hai }]-[\text { hau }]\end{array}$ \\
\hline Engl. & [ji:ld $]-\quad[w i: l d]$ & $\begin{array}{l}\text { Hai hau } \\
\text { [hai]- [haun] }\end{array}$ \\
\hline & $\begin{array}{l}\text { yield wield } \\
\text { ergeben' führen" }\end{array}$ & high how \\
\hline Frz. & 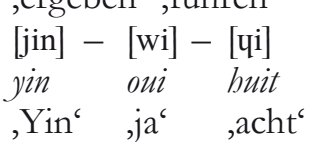 & \\
\hline
\end{tabular}

In Übereinstimmung mit der Sonoritätsskala in (5) zeigen diese Daten, dass als einziger Vokal in einer Randposition nur /i/ erscheint; treten dort zwei Vokale auf, erscheinen /i/ und /u/. Der Umstand, dass diese beiden Vokale im Englischen und im Deutschen in der Koda nur nach /a/ kontrastieren, weist auf besondere Neutralisationsmuster hin. In Tabelle (42) wird das Kontrastpotenzial im Nukleus vor den fraglichen Kodavokalen in den beiden Sprachen verglichen:

a. Deutsch

b. Englisch

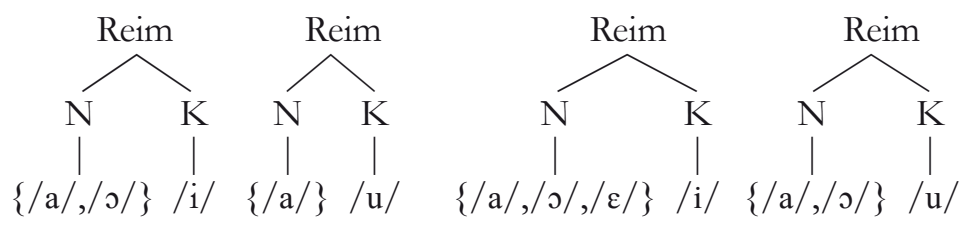

Die relevanten Kontraste sind in (43) veranschaulicht:

$\begin{array}{lll} & \text { Kontraste vor Koda-/i/ } & \text { Kontraste vor Koda-/u/ } \\ \text { Deutsch } & {[\text { hai }]-[\mathrm{hoi}]} & {[\mathrm{hau}]} \\ & H a i \text { Heu } & \text { hau } \\ \text { Englisch } & {[\mathrm{sai}]-[\mathrm{ssi}]-[\mathrm{sri}]} & {[\mathrm{hou}]-[\mathrm{hau}]} \\ & \text { sigh soy say } & \text { hoe how }\end{array}$


Auch die Neutralisationsmuster in (43) bestätigen die Sonoritätsabstufungen in (5): Eine universelle Präferenz für ein möglichst ausgeprägtes Sonoritätsgefälle im Reim ließe erwarten, dass das Kontrastpotenzial im Nukleus je stärker beschränkt ist, desto sonorer der Kodavokal ist. Diese Generalisierung bliebe in der alternativen Analyse in (44) unberücksichtigt, wo das Kontrastpotenzial im Nukleus unabhängig von der Besetzung der Koda immer gleich ist (Ramers/Vater 1995, S. 109; Hall 1992, S. 140):

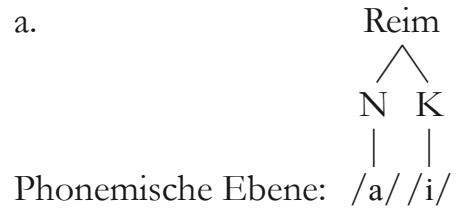

Phonetische Ebene:

[ai]

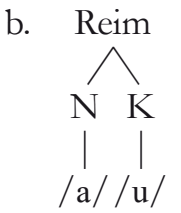

[aun]

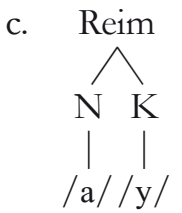

[oi $]$

Die phonologische Evidenz für die Analyse betrifft sprachinterne Alternationen wie in $M[\mathrm{au}]$ s Maus - M[oi] se Mäuse, die sich unter der Annahme der Repräsentation in (44c) analog zu den Alternationen in $F[\mathrm{u}:] \beta F u \beta-F[\mathrm{y}:] \beta e$ Füße durch eine einfache „Fronting“-Regel ([+hinten] $\rightarrow$ [-hinten]) beschreiben ließen. Weitere Beispiele zeigen aber, dass Umlaut auch eine „Hebung“ des Vokals im Nukleus beinhalten kann (z.B. B[a]ll Ball-B[ع]lle Bälle), eine Regel, die möglicherweise auch die [au - [oi]-Alternation umfasst. So beinhalten Vokalalternationen in deutschen Indikativ-Verbparadigmen notwendig Hebung (siehe (45a)), niemals „Fronting“ (siehe (45b)). Das Auftreten der [au ] - [oi]-Alternationen in (45c) lässt entsprechend darauf schlieBen, dass auch diese Alternation Hebung (d.h. /a/ to / / im Nukleus, vgl. (42a)), anstatt „Fronting“" (d.h. /u/ to /y/ in der Koda, vgl. (44)), beinhaltet.

$$
\begin{aligned}
& \text { a. } \mathrm{n}[\mathrm{e}:] \text { hme }-\mathrm{n}[\mathrm{I}] \mathrm{mmst} \\
& \text { s[e:]he }-\mathrm{s}[\mathrm{i}:] \text { hst } \\
& \mathrm{w}[\mathrm{a}] \mathrm{sche}-\mathrm{w}[\varepsilon] \text { schst } \\
& \text { c. } 1[\mathrm{au}] \mathrm{fe}-1[\mathrm{oi}] \mathrm{fst} \\
& \mathrm{s}[\mathrm{au}] \mathrm{fe}-\mathrm{s}[\mathrm{ji}] \mathrm{fst}
\end{aligned}
$$
b. $\quad r[u:] f e-r[u:]$ fst $\left(*_{r}[y:] f s t\right)$ $\mathrm{k}[\mathrm{\jmath}] \mathrm{mme}-\mathrm{k}[\mathrm{\jmath}] \mathrm{mmst}(* \mathrm{k}[œ] \mathrm{mmst})$$$
\mathrm{t}[\mathrm{u}:]-\mathrm{t}[\mathrm{u}:] \mathrm{st}\left({ }^{*} \mathrm{t}[\mathrm{y}:] \mathrm{st}\right)
$$

Ein zweites phonologisches Argument für die phonemische Analyse von [oi] als / oi / statt / ay/ betrifft die phonotaktischen Beschränkungen in (46). Im Deutschen erscheint der palatale Approximant [j] nie vor den Diphthongen [ai] oder [oi], kombiniert aber mit [au ], (jauchzen, Jauche, Kabeljau, jaulen, Jause). Unter der Annahme der phonemischen Repräsentationen in (46) lässt sich diese Lücke mit Bezug auf eine Markiertheitsbeschränkung gegen Silben erklären, in denen der Nukleus von gleichen Segmenten in Ansatz und Koda flankiert wird (siehe das „Shell Law“ in Vennemann 1988, S. 11). 


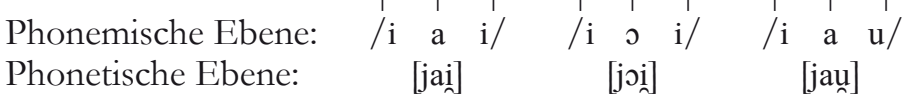

Das wohl wichtigste Argument für die Bestimmung der phonemischen Repräsentation betrifft die Ableitbarkeit der beobachtbaren phonetischen Variation. Unter Annahme der Phonemfolge /oi/ wäre die lautliche Beschaffenheit des Erstglieds [0] bereits erklärt; die Variation im Zweitglied ließe sich als Modifikationen der beteiligten artikulatorischen Gesten plausibel ableiten (siehe Abschnitt 4). Anders verhielte es sich bei Annahme der Folge /ay/: dass ein hoher, runder, unsilbischer Vokal keine entsprechende Assimilation des vorangehenden Vokals bewirkt, zeigt die Aussprache des Diphthongs [aun], wo das Erstglied /a/ weder gehoben noch gerundet erscheint.

Die Annahme, dass die so genannten Diphthonge im Deutschen und Englischen durch die Kodasilbifizierung hoher Vokoide bedingt sind, wird durch das Verhalten intervokalischer hoher Vokoide bestärkt. Im Englischen erscheint intervokalisches / i/ stets in der Koda (vgl. (47a)), es sei denn, ein hoher gespannter Vokal geht voran (vgl. (47b)):

a.

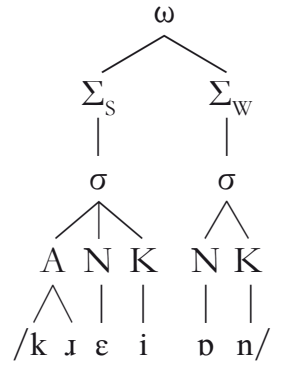

$\left[\mathrm{k}^{\mathrm{h}} . \mathrm{i \varepsilon i} \cdot{ }_{n}^{\mathrm{j}} \cdot \mathrm{pn}\right]$

crayon, Stift $^{6}$ b.

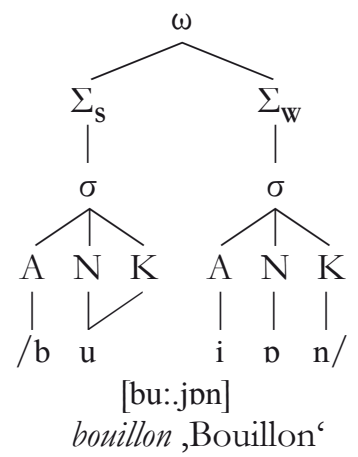

Auch im Deutschen wird die Silbifizierung von postvokalischem /i/ gewöhnlich durch den Kontext bestimmt. Wie im Englischen erscheint das /i/ meist in der Koda (vgl. (48a/b)), es sei denn ein Vollvokal folgt: In diesem Fall erscheint es im Ansatz (vgl. (48c)).

Die Strukturen in (48) veranschaulichen die im Deutschen ausnahmslos geltende Regel, dass gespannte Vokale auf der phonemischen Ebene nur in offenen, ungespannte Vokale nur in geschlossenen Silben erscheinen, wobei /i/ in der Koda die in (42a) beschriebene Neutralisation im Nukleus bewirkt. Die Silbifizierungsmuster in (48) sind nur in wenigen Wörtern ver- 
letzt, wobei weiterhin gilt, dass gespannte Vokale nur in offenen, ungespannte nur in geschlossenen Silben erscheinen (z.B. /i/ vor Schwa im Ansatz in /ko.iə/ [kº́:.jə] Koje, /fø.iə.tõ/ [fǿ:.jə.th õ ] Feuilleton; /i/ vor Vollvokal in der

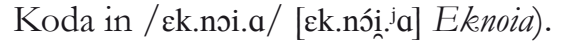

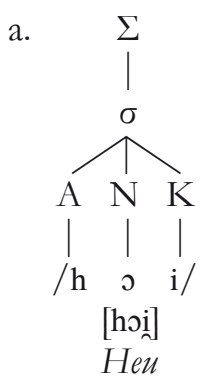

b.

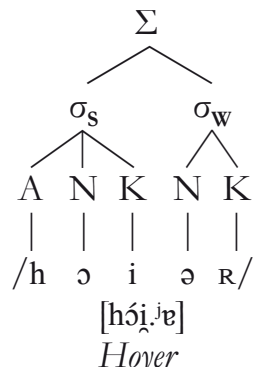

c.

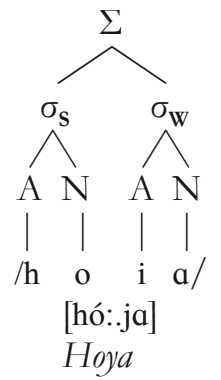

Im Französischen erscheinen intervokalische Vokoide immer im Ansatz (siehe (49a)). Vor Konsonant sind hohe Vokoide hingegen stets silbisch und ergeben somit nach Vokal einen Hiatus (siehe (49b)). Unter keinen Umständen sind solche Vokale als Koda silbifizierbar: maïs kann nicht als eine Silbe gesprochen werden, im Gegensatz zu Wörtern mit vergleichbarer phonemischer Struktur im Deutschen oder Englischen (vgl. deutsch Mais, englisch mice ,Mäuse').
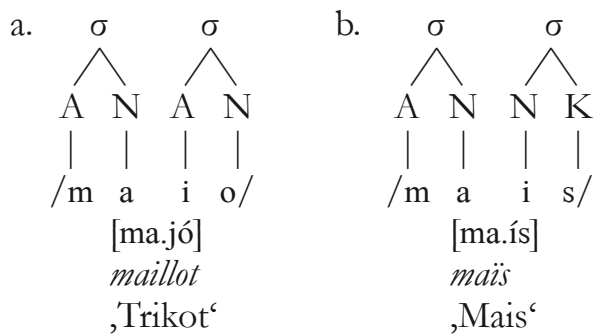

Die Generalisierung, dass das Französische im Gegensatz zum Deutschen oder Englischen keine „Diphthonge“ hat, lässt sich entsprechend als Folge einer Beschränkung gegen die Organisation hoher Vokoide in der Kodaposition erklären. Der hier relevante Unterschied zwischen den Sprachen beträfe dann nicht die Phoneminventare, sondern die Silbifizierungsregeln. Auch das Auftreten von [j] am Wortende wie in [baj] bail ,Pachtvertrag ${ }^{6}$ motiviert nicht die Annahme eines eigenen Phonems, da auch hier kein Kontrast mit silbischem [i] möglich ist. Vielmehr zeigt sich hier eine für das Wortende charakteristische Besonderheit, die darauf hindeutet, dass das wortfinale Phonem den Ansatz einer so genannten katalektischen Silbe, d.h. einer Silbe mit leerem Nukleus, bilden kann wie in (50a) (siehe Harris/ Gussman 2002). Die fraglichen Besonderheiten, und mit ihnen die Motiva- 
tion der katalektischen Silben, betreffen ausschließlich das Kontrastpotenzial auf der phonemischen Ebene; in den entsprechenden phonetischen Repräsentationen sind wortfinale unsilbische Segmente als Koda zu organisieren (siehe (50b)).
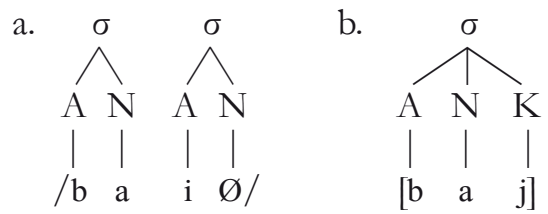

Die Annahme, dass im Französischen wortfinales /i/ nach Vokal auf der phonemischen Eben nicht als Koda sondern als Ansatz silbifiziert wird, erklärt auch das große Kontrastpotenzial im vorangehenden Nukleus. Die Tabelle in (51) zeigt, dass vor wortfinalem [j] die Kontrastmöglichkeiten ähnlich ausgeprägt sind wie vor prävokalischem [j]. Die Verteilung unterscheidet sich damit deutlich von den Beschränkungen zu möglichst sonoren Vokalen, die im Deutschen und Englischen vor /i/ in der Koda beobachtet werden können (vgl. (42)).

\begin{tabular}{|c|c|c|c|c|c|c|c|c|c|c|c|}
\hline & $/ \mathrm{i} /$ & $/ \mathrm{u} /$ & $/ \mathrm{y} /$ & $/ \mathrm{e} /$ & $/ \mathrm{e} /$ & $/ \mathrm{o} /$ & $/ \mathrm{o} /$ & $/ \varnothing /$ & $/ \mathrm{e} /$ & $/ \mathrm{a} /$ & $/ \mathrm{a} /$ \\
\hline -[j]\# & $\checkmark$ & $\checkmark$ & - & & $\checkmark$ & & $(\checkmark)$ & & $\checkmark$ & $\checkmark$ & $\checkmark$ \\
\hline -[j] $\mathrm{V}$ & $\checkmark$ & $\checkmark$ & $\checkmark$ & $\checkmark$ & & $(\checkmark)$ & & $\checkmark$ & $\checkmark$ & $\checkmark$ \\
\hline
\end{tabular}

Die Distribution der Vokale in (51) ist in (52) veranschaulicht:
a. [baj] bail
,Pachtvertrag
[vje:j] vieil
,alt ${ }^{6}$
[गR.gœ:j] orgueil
,Hochmut
[gRə.nuj] grenowille
,Frosch
[fij] fille
b. [ba.jœ:R] bailleur
,Pächter
[vje.ji:R] vieillir
,altern
[गR.gœ.jø] orgueilleux
,hochmütig'
[gRə.nu.jz:R] grenouillère
,Froschteich“
,Tochter
[fi.jet] fillette
,Töchterchen`

Im Gegensatz zum Französischen bildet finales /i/ im Deutschen nie den Ansatz einer katalektischen Silbe, sondern schließt nach Vokal gewöhnlich die Silbe (vgl. (48a)). In den Ausnahmefällen, wo die Kodasilbifizierung aufgrund eines vorangehenden gespannten Vokals ausgeschlossen ist, bildet das wortfinale /i/ den Nukleus (vgl. (53a)). 


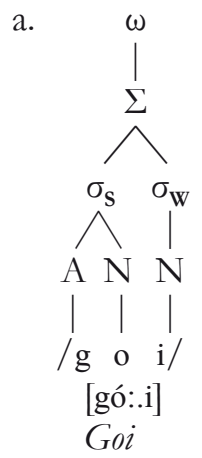

b.

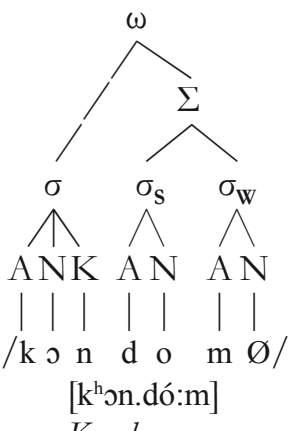

c.

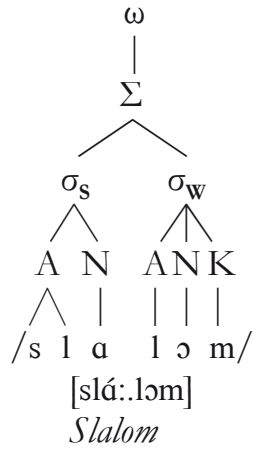

Die Annahme, dass wortfinale Konsonanten auch im Deutschen den Ansatz katalektischer Silben bilden können wie in (53b), ist durch eine mit dem Wortende verknüpfte Besonderheit begründet: Wortintern kommen gespannte Vokale in geschlossenen Silben nicht vor (vgl. *K[o]n.dom); ihr scheinbar regelwidriges Auftreten vor dem wortfinalen Konsonanten erklärt sich durch dessen Silbifizierung als Ansatz. Auch der Zusammenhang zwischen Vokalgespanntheit und Betonung wäre somit erklärt: Nimmt man an, dass das Auftreten eines gespannten Vokals vor dem wortfinalem Konsonanten eine katalektische Silbe bedingt wie in (53b), und dass die letzten beiden Silben im Wort im Regelfall einen trochäischen Fuß bilden, so fällt der Wortakzent auf die zweite Silbe in Kondóm, aber auf die erste Silbe in Slálom. ${ }^{32}$

Die Annahme, dass wortfinales [j] in den französischen Wörtern in (52a) auf der phonemischen Ebene als Ansatz einer katalektischen Silbe und nicht als Koda organisiert ist, ist wichtig für den Sprachvergleich in Tabelle (40). Insbesondere ist diese Analyse eine Voraussetzung für die Aufrechterhaltung der Generalisierung, dass die Inventare der angeblichen Diphthonge im Deutschen und Englischen lediglich als Folge einer beschränkten Verteilung der Vokale im Nukleus zu betrachten ist, die sich aufgrund der hohen Sonorität der Phoneme in der Koda ergibt. Diese Analyse wird durch die in (54) veranschaulichten Neutralisationsmuster im Amerikanischen Englischen unterstützt. Im Gegensatz zu den Vokalfolgen in (42) werden die fraglichen Lautfolgen in (54) nie als Diphthonge bezeichnet; die Beschränkungen hinsichtlich des Kontrastpotenzials im Nukleus sind dennoch ähnlich. Wie vor deutsch /i/, kontrastieren auch vor englisch $/ \mathrm{r} /$ in der Koda nur zwei Vokale im Nukleus: /a/ und / $/$.

$32 \quad$ Dass ein gespannter Vokal nicht inhärent den Akzent auf sich zieht, zeigt sich in der Stabilität des Akzents auf dem ungespannten Vokal in der pänultimalen Position in Wörtern wie Madónna gegenüber Wörtern mit gespanntem Vokal wie Altóna Áltona: Auch in dieser Position ist die Silbenstruktur maßgeblich (geschlossen versus offen; siehe Vennemann 1994, S. 19). 
$\mathrm{f}[\mathrm{a.I}] \mathrm{mer}$ farmer st[a..]k stark

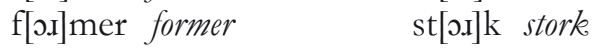

Das Neutralisationsverhalten in (54) hängt vermutlich damit zusammen, dass im Englischen / $\mathrm{r} /$ auch als Nukleus erscheinen kann und mag letztlich durch die erheblich offenere Artikulation von Englisch [r], verglichen mit dem Deutschen oder Französischen [R], bedingt sein. Die Neutralisationsmuster in (54) sind ebenfalls nur vor wortfinalem /r/ verletztbar (vgl. b/rr/ beer, $\mathrm{b} / \mathrm{er} /$ bear, $\mathrm{b} / \mathrm{vr} /$ boor $)$, ein Hinweis auf Katalexis im Englischen. ${ }^{33}$

\section{Phonetik}

Die Behauptung, dass notwendig unsilbisches [j] wie in [j] a ja, potenziell silbisches [i] wie in Foli]e Folie, notwendig silbisches [i] wie in $M[\mathrm{i}] m o s e$ Mimose, und schließlich das notwendig unsilbische Zweitelement schlieBender Diphthonge wie in $\mathrm{Ha}$ [i] Hai allesamt Allophone des Phonems /i/ sind, wirft die Frage auf, wie die teils gravierenden phonetischen Unterschiede zwischen diesen Lauten zu erklären sind. In Abschnitt 4.1 wird zunächst der Zusammenhang zwischen den spezifischen phonetischen Eigenschaften der Allophone und den jeweiligen Silbenpositionen Nukleus, Ansatz und Koda erläutert. In Abschnitt 4.2 werden die Besonderheiten von /i/-Allophonen behandelt, die im Deutschen als Zweitglied in einem komplexen Ansatz erscheinen. Schließlich werden in 4.3 /i/-Allophone behandelt, die als Nukleus vor einem weiteren Nukleus erscheinen.

\subsection{Allophonie als Funktion von Silbenpositionen}

Geht man davon aus, dass Phoneme bestimmte artikulatorische sowie perzeptuelle Zielstrukturen enkodieren und dass /i/ ein geschlossener Vorderzungenvokal ist, so lässt sich die beobachtbare phonetische Variation als Funktion der unterschiedlichen Silbenstrukturen ableiten. Die relevanten Muster sind in (55) grob skizziert, wobei die Linien die charakteristische Variation - bzw. Konstanz - hinsichtlich der Zungenhöhe repräsentieren.
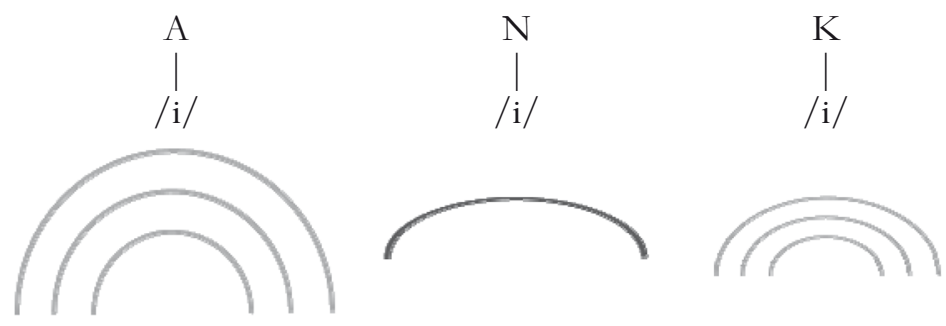

33 Die einzigen Ausnahmen enden auf /d/ oder /s/, zwei Konsonanten, die häufig als Suffix auftreten (beard, weird, fierce, pierce, scarce). 
Die geringste Variation findet sich in der Nukleusposition, wo, bedingt durch Betonung, vor allem Dauerunterschiede feststellbar sind. So ist das unbetonte /i/ in Mimóse wesentlich kürzer als das /i/ in Mime, das in der ersten Silbe des Fußes auftritt:

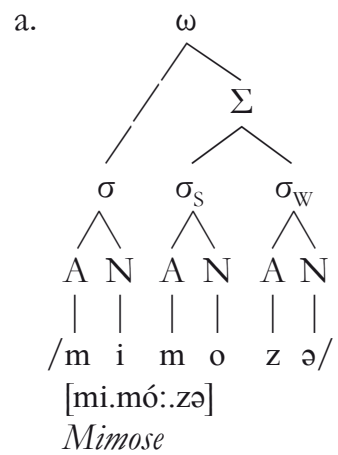

b.

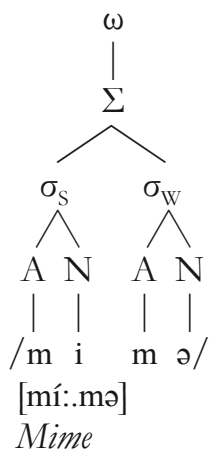

Die Aussprache von /i/ in der Ansatzposition charakterisiert Kohler (1995, S. 156) wie folgt:

$/ \mathrm{j} /$ ist ein palataler Gleitlaut, der im allgemeinen als stimmhafter Approximant realisiert wird und sich schnell aus einer hohen vorderen Vokoidposition vom Typ [i] in die Position für den nachfolgenden Vokal begibt.

Generell ist die Artikulation von /i/ in der Ansatzposition durch ein schnelles Absenken der Vorderzunge zwecks Aussprache des nachfolgenden Vokals bestimmt: Ist dieser Vokal offen, so reicht bereits eine mittlere Zungenhöhe, um diesen Effekt zu erreichen. Folgt ein geschlossener Vokal, insbesondere ein /i/, so ist eine entsprechend höhere Position erforderlich, um das für den Gleitlaut charakteristische Absenken der Zunge auszuführen. Die entsprechenden Unterschiede in der Zungenhöhe in den Vergleichssprachen beschreibt Jespersen (1913, S. 48 f.) wie folgt:

Auch das englische [j] ist je nach der Umgebung verschieden, obgleich die Unterschiede nicht so ausgeprägt sind wie im Französischen; in yard [ja:d] ist es entschieden mehr vokalisch als in yet [jet] oder gar in yield [ji:ld].

Die Daten in (57) (Brinkmann i.Vorb.) zeigen, dass die für Gleitlaute charakteristische Abhängigkeit der Zungenhöhe vom adjazenten Vokal auch im Deutschen feststellbar ist. Insbesondere zeigt sich, dass potenzielle Friktion im Deutschen von der Zungenhöhe des Folgevokals abhängt: Folgt ein offener Vokal, so ist Friktion selten, folgt ein hoher Vokal, so steigt der Anteil der Aussprachen mit Friktion auf über 50 Prozent.

Generell gilt, dass die silbeninitiale Position, insbesondere wenn es sich gleichzeitig um die fuß- oder wortinitiale Position handelt, eine phonetische Stärkeposition ist. Das Auftreten von Friktion kann dabei als Folge beson- 
ders stark ausgeführter artikulatorischer Gesten aufgefasst werden, wo unter Umständen sogar über das Ziel hinausgeschossen wird (Browman/Goldstein 1995).

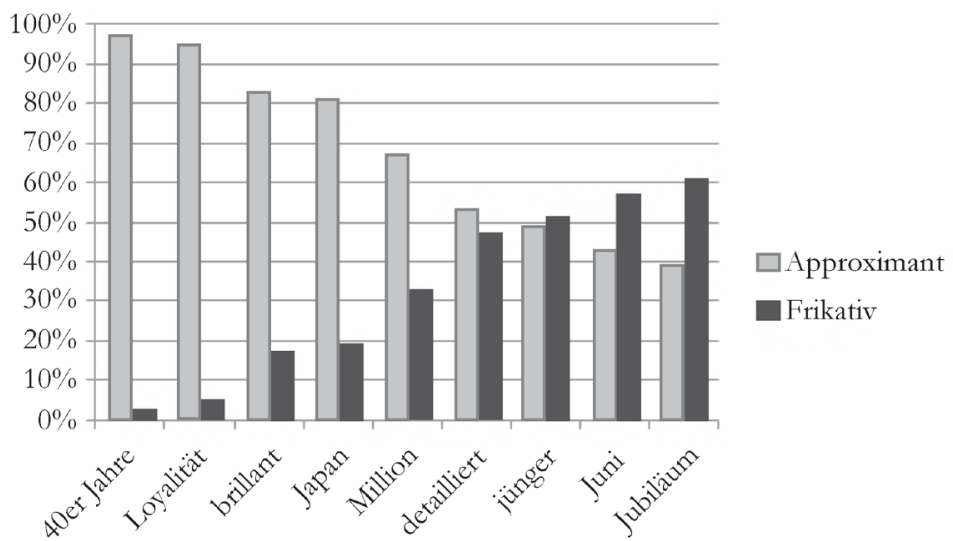

Die Darstellung der / $\mathrm{i}$-Allophonie in der Kodaposition in (55) entspricht der in Tabelle (58) aufgeführten Variation in der Transkription der so genannten schließenden Diphthonge in deutschen Aussprachewörterbüchern. Während die jeweiligen Erstglieder von allen Autoren gleich transkribiert werden, findet sich erhebliche Variation in den Umschriften der unsilbischen Bestandteile der Diphthonge:

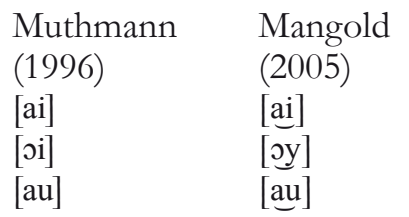

$\begin{array}{ll}\text { Krech et al. } & \text { Siebs } \\ (2009) & (1957) \\ {[\mathrm{a} \varepsilon]} & {[\mathrm{ae}]} \\ {[\mathrm{o}]} & {[\mathrm{o}]} \\ {[\mathrm{a}]} & {[\mathrm{ao}]}\end{array}$

Die hier zu sehende Variation ist charakteristisch für die Silbenkoda, eine Schwächeposition, in der das artikulatorische Ziel, d.h. /i/ bzw. /u/, eher nicht erreicht wird. ${ }^{34}$ Eine ähnliche Variation in der Aussprache der Diphthonge wird auch für das Englische beschrieben (Eckert/Barry 2002, S. 157). Die phonetischen Beschreibungen lassen demnach den Schluss zu, dass deutsche und englische schließende Diphthonge, trotz möglicher Unterschiede in der phonetischen Realisierung, als biphonemische Sequenzen mit finalem /i/ bzw. /u/ analysierbar sind. Entscheidend ist, dass die jeweilige phonetische Variation in den Streubereich fällt, der bei Annahme dieser Phoneme in einer durch verminderte artikulatorische Anstrengung charakterisierten prosodischen Position erwartbar ist.

$34 \quad$ Die Symbole für gerundete Vokale, die von den meisten Autoren für den Diphthong / oi/ gewählt werden, reflektieren demnach lediglich die assimilatorische Rundung, die durch den Vokal /o/ begründet ist. 
Der Wert der in (55) skizzierten allophonischen Analyse liegt somit in der Herstellung eines Zusammenhangs zwischen unabhängig begründeten prosodischen Positionen ${ }^{35}$ und universell mit diesen Positionen assoziierten artikulatorischen Ausführungsmodi bei gleichzeitiger Reduktion des Phoneminventars. Das heißt, sämtliche phonetische Unterschiede zwischen dem (möglichen) phonetischen Frikativ vor hohem Vokal in [j] uni Juni, dem Gleitlaut vor nichthohem Vokal in [j]a ja, dem langen [i:] in $n[\mathrm{i}:]$ nie, und dem mittleren, gerundeten unsilbischen Vokal $[\varnothing]$ in $n[ø ø]$ neu sind als kontextuell bedingte Modifikationen von Stärke und Dauer der mit dem Phonem /i/ verknüpften artikulatorischen Gesten ableitbar. Ebenso sind die phonetischen Unterschiede zwischen dem ersten und letzten Segment in deutsch Robr in (59a) mit der Annahme eines Phonems vereinbar (Shannon 1984, S. 235). Auch hier lässt sich nachweisen, dass die Unterschiede zwischen dem wortinitialen $[\mathrm{b}]$, das durch mögliche Friktion gekennzeichnet ist, und dem finalen unsilbischen [ํ], einem offenen Vokal, als für Ansatz versus Kodaposition charakteristischen Modifikationen der mit dem Phonem / R/ verknüpften Gesten erklärbar sind (Schiller/Mooshammer 1995). ${ }^{36}$

a. Deutsch

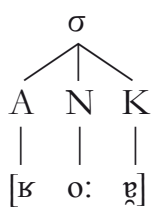

Rohr b. Französisch

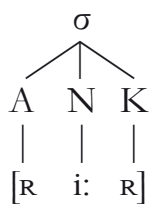

rire

,lachen ${ }^{6}$

Dass phonetische Fortisierungs- bzw. Lenisierungsprozesse nicht nur phonem- sondern auch sprachspezifisch sind, zeigt der Vergleich mit dem Französischen [Ri:R] in (59b), wo die fraglichen / $\mathrm{R} /$-Allophone eher geringe durch Silbenpositionen bedingte Variation aufweisen.

\section{2 /i/ als Zweitglied in einem komplexen Ansatz}

Die Silbifizierung von /i/ als Teil eines wortinternen komplexen Ansatzes bedingt erhebliche Variation. Das Wort Furie etwa kann mit konsonantischem [r] und silbischem [i], aber auch mit vokalischem [] und unsilbi-

\footnotetext{
35 Die prosodische Struktur ist durch die Abfolge und Sonorität der beteiligten Phoneme begründet.

36 Auf der phonemischen Ebene bildet der finale Konsonant in deutsch Rohr nach gespanntem Vokal den Ansatz einer katalektischen Silbe. Das Wort verletzt daher nicht die Markiertheitsbeschränkung gegen identische Konsonanten in Ansatz und Koda (vgl. (46)).
} 
schem [j] ausgesprochen werden (vgl. Kohler 1995, S. 157). Diese Zusammenhänge legen eine Darstellung der phonetischen Repräsentationen wie in (60) nahe:

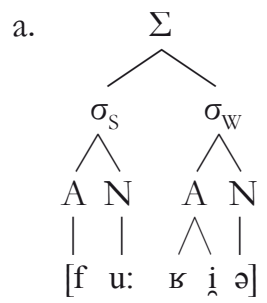

b.

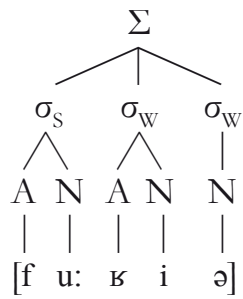

c.

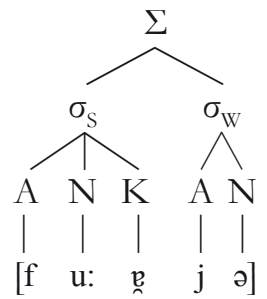

Ein grundsätzliches Problem bei prosodischen Darstellungen phonetischer Repräsentationen besteht darin, dass prosodische Strukturen kategorisch sind, phonetische Realisierungen aber inhärent gradient sind. So ist unklar, wie etwa Mangolds Generalisierung, dass [i] nach [r] vor unbetontem Vokal „nicht so leicht" unsilbisch wird wie vor betontem Vokal (2005, S. 42), dargestellt werden kann. An dieser Stelle soll lediglich darauf hingewiesen werden, dass /i/ im wortinitialen komplexen Ansatz immer unsilbisch gesprochen wird (z.B. [fjel] Fjäll), wortintern aber stark zwischen silbischen und unsilbischen Aussprachen variiert, wobei die Silbischkeit selbst oft zweifelhaft ist. ${ }^{37}$ Die Annahme des komplexen Ansatzes auf der phonemischen Ebene wie in (37c) ist dabei vor allem durch die Neutralisation der Gespanntheitsopposition in der betonten Silbe bedingt: Wenn /R/ und /i/ als komplexer Ansatz organisiert werden, ist die betonte Silbe notwendig offen, so dass nur gespannte Vokale erscheinen können (d.h. F/u./ rie, nicht ${ }^{*} \mathrm{~F} /$ v./ rie).

\subsection{Phonetische Gleitlautbildung}

Die Wörter Liane und liiere sind beide dreisilbig im Deutschen. Sie unterscheiden sich darin, dass der Hiat in Liane durch einen Gleitlaut gekennzeichnet ist, wobei die Zunge im Übergang von /i/ zu dem offenen Vokal zunächst kurz angehoben und dann gesenkt wird. Die Funktion dieser Bewegung könnte in einer Verbesserung der phonetischen Silbenstruktur bestehen, indem das /i/ einen Ansatz für die Folgesilbe lizensiert wie in (61b). Die auf der phonemischen Ebene festgelegte Assoziation mit dem Nukleus (vgl. 61a) bleibt dabei erhalten. ${ }^{38}$

$37 \quad$ Diese Variation findet sich auch in den Aussprachewörterbüchern. Besonders Krech et al. (2009) transkribieren zuweilen silbisches [i], wo andere unsilbisches [i] haben (z.B. Salmiak, Hernie). Nur Siebs (1957) transkribiert silbisches [i] zwischen /R/ und unbetontem Vollvokal (Gloria, Karies).

38 Wörter wie Piano oder Fiasko werden demnach im Deutschen trotz phonetischer Gleitlautbildung dreisilbig ausgesprochen, im Gegensatz zu den jeweiligen Entsprechungen im Französischen. 
a.

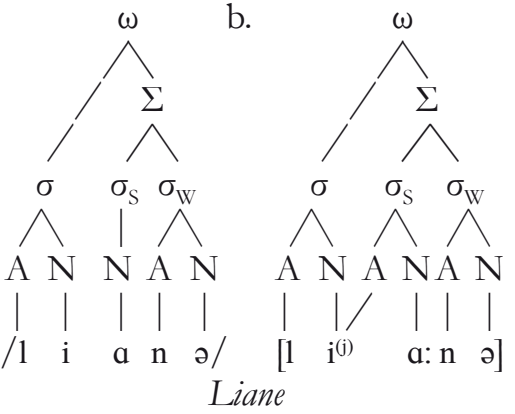

c.

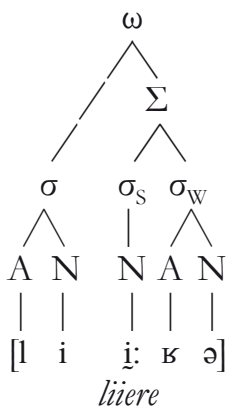

Die Annahme, dass /i/ nur vor sonorerem Folgevokal einen Ansatz lizensiert, erklärt die unterschiedliche Glottalisierbarkeit der betonten Vokale in $(61 \mathrm{~b} / \mathrm{c})$ : Dieser Fortisierungsprozess tritt nur in strikt wort- oder fußinitialer Position auf. ${ }^{39}$ Auch das betonte /i/ in Ruine erscheint somit glottalisiert, das betonte /a/ in Ruanda hingegen nicht. ${ }^{40}$

\section{Lexikalische Ausnahmen}

Bislang wurde argumentiert, dass [j], [i] und [i] sich als durch unterschiedliche prosodische Positionen bedingte /i/-Allophone analysieren lassen, wobei sich die fraglichen Positionen aufgrund des Kontexts, insbesondere der jeweiligen Phonemfolgen, ergeben. Die Beschreibung knüpft somit an strukturalistische Modelle sowie die Unterscheidung der Ebenen in der Lexikalischen Phonologie an, wo die wichtigen Kriterien wie folgt gegenübergestellt worden sind (siehe Libermann 1983):

Phonologie

Betrifft die prosodische

Organisation von Phonemen: diskret

Betrifft das Kontrastpotenzial

Lexikalische Ausnahmen möglich
Phonetik

Betrifft Modifikationen artikulatorischer Gesten: gradient

Betrifft die Implementation

Keine lexikalischen

Ausnahmen

39 Eine alternative Erklärung des Glottalisiersunterschieds mit alleinigem Bezug auf die betonten Vokale scheidet aus, da tiefe Vokale inhärent eher zu Glottalisierung neigen als hohe Vokale (siehe Krech 1968).

40 Mangold (2005) transkribiert [ru'i:nə] versus ['ruanda]. Es zeigt sich, dass alle hohen, optional sogar mittlere Vokale, vor sonorerem Folgevokal einen Ansatz lizensieren. So glottalisieren manche Sprecher das betonte $[\mathrm{a}]$ in Theáter nicht. Betonte Vokale nach / $\mathrm{a} /$ sind immer glottalisierbar. 
Der letzte Punkt verdient besondere Beachtung, weil lexikalische Ausnahmen oft als Evidenz für Minimalpaare betrachtet werden. So beweisen Wörter wie [bj] örn Björn vs. [bi] ánca Bianca nicht die Notwendigkeit, zwei getrennte Phoneme /i/ und / $\mathrm{j} /$ anzuerkennen. Vielmehr zeigen sie, dass die Silbifizierung von Phonemen Ausnahmen haben kann: Gewöhnlich bildet /i/ nach wortinitialem Konsonanten einen Nukleus wie in Bianca; in Björn ist die Ansatzsilbifizierung von /i/ im Lexikon festgelegt. Möglicherweise ist diese Ausnahme durch die Entlehnung der schriftlichen Form und der im Deutschen bestehenden Verknüpfung des Graphems $<j>$ mit einer Ansatzsilbifizierung bedingt. Schriftaussprachen sind auch als Gründe für die Nukleus- statt Ansatzsilbifizierung von wortinitialem /i/ vor Vokal in Fällen wie Deutsch Iota, Iambus zu nennen, wo die Schreibweise jeweils aus dem Griechischen entlehnt wurde (vgl. die Umschriften [i|ó:ta] und [i|ámbus] in Siebs 1957). Die letzteren Beispiele veranschaulichen auch die charakteristische Instabilität, die lexikalische Ausnahmen von echten Minimalpaaren unterscheidet: die neuen Varianten Jambus und Jota sind Belege für Regularisierung von Ausnahmen (vgl. die Umschriften [jó:ta] und [jámbus] in Siebs 1957). ${ }^{41}$

Regularisierung ist nicht zu erwarten, wenn Regelverletzungen erwünscht sind wie in Interjektionen. Die Beispiele in (63a) verletzen allgemeine Regeln für die Zuordnung von Phonemen und Silbenpositionen im Deutschen. Bei den Beispielen in (63b) geht es um Regelverletzungen im Zusammenhang mit dem Phonem /i/. Die Silbifizierung von /i/ nach wortinitialem Konsonanten wie in Tja! oder Njam Njam! im Ansatz ist dabei als Ausnahme zu betrachten, die bestätigt, dass ein solches /i/ regulär als Nukleus zu silbifizieren ist. Die Beispiele in (63c) zeigen, dass die Interjektionen in den Vergleichssprachen ähnliche Verletzungen zeigen.

Interjektion

a. /pst/ Pst! /zo/So!

b. /hui/ Hui /tia/ Tja!

c. Engl. /uau/Wow Frz. /ui/ Ouille!

\section{Regelverletzung}

Kein vokalischer Nukleus

Ungespannter Vokal in offener Silbe /v/ vor /i/ im Nukleus

/i/ nach wortinitialem Konsonanten im komplexen Ansatz

Identische Segmente in Ansatz und Koda Nukleus-Ansatz Silbifizierung (statt Ansatz-Nukleus, vgl. /ui/ oui ,ja)

${ }_{41}$ Auch die angeblichen Kontraste in Bill[i] on Billion versus Bill[j] et Billet oder Mill[i] arde Milliarde versus Bill[j] ard Billard (vgl. Siebs 1957) können als Beispiele für Schriftaussprache gelten. Empirisch lassen sich diese Kontraste allerdings nicht bestätigen (vgl. die Ergebnisse in (57)). Moultons Entschluss, aufgrund dieser vermeintlichen Minimalpaare auf eine allophonische Analyse zu verzichten und ein getrenntes Phonem / $\mathrm{j} /$ anzuerkennen, ist daher unmotiviert (Moulton 1962, S. 65). 
Regelverletzungen wie in (63) sind von Fällen zu unterscheiden, in denen die prosodische Organisation von Phonemen durch morphologische Grenzen beeinflusst ist. So ist die Silbischkeit von /i/ in $L a[. i$.$] zísmus, verglichen$ mit Ta[i] fún Taifun, durch die unterschiedlichen morphologischen Strukturen (in (64) durch eckige Klammern markiert) und sich daraus ergebenden Silbifizierungsdomänen der Wörter (in (64) durch runde Klammern markiert $)$ zu erklären $(\mathrm{W}=$ Wort, $\mathrm{ST}=\mathrm{Stamm}, \mathrm{SF}=$ Suffix $)$ :
a. $[\text { taifun }]_{\mathbb{W}}$
b. (taifun)
$\left[[\mathrm{la}]_{\mathrm{ST}}[\mathrm{its}]_{\mathrm{SF}}[\mathrm{Ism}]_{\mathrm{SF}}[\mathrm{Us}]_{\mathrm{SF}}\right.$
(la) (itsismus)

Hinsichtlich der morphologischen Struktur ist anzumerken, dass diese sprachübergreifend in erster Linie durch die Erkennbarkeit von Affixen determiniert ist. So lässt sich in Laizismus analog zu Wörtern wie Stoizismus oder Romantizismus die Suffixfolge ,its+ism+us' erkennen. Hinsichtlich der Silbifizierungsdomänen ergibt sich die weitere sprachübergreifende Generalisierung, dass vokalinitiale Suffixe regulär in die Domäne des vorangehenden Morphems integriert werden, falls dieses auf einen Konsonanten endet. Die Vokale der Suffixe -ism und -us erscheinen somit nicht in silbeninitialer Position, im Gegensatz zu dem Vokal in dem Suffix -its, das auf einen Vokal folgt. Folglich erscheint es in der initialen Position der Silbifizierungsdomäne (vgl. (64b)) und bildet daher einen Nukleus (La[.i.] zísmus). ${ }^{42}$

Zusammenfassend lässt sich sagen, dass es im Deutschen keine Kontexte gibt, wo notwendig oder potenziell silbische mit nichtsilbischen vorderen hohen Vokoiden kontrastieren. Es gibt hingegen einige Kontexte, wo die Verteilung dieser Laute regulär durch die phonologische Umgebung gesteuert ist, wie etwa das Ausgeschlossensein von silbischem [i] nach /1/ oder /n/, denen ein kurzer betonter Vokal vorangeht (*Kón[i.]ak Konjak), oder das Augeschlossensein von nichtsilbischem [j] nach Konsonantenfolgen mit stark steigender Sonorität (Schlendr[j]an Scblendrian). Die Verteilung der Laute kann daher als allophonisch bezeichnet werden; das zugrundeliegende Phonem ist /i/.

\section{Schluss}

Die vorliegende Studie ist dem Nachweis gewidmet, dass im Deutschen der palatale Approximant wie in $j a$ und das Zweitglied der Diphthonge wie in Hai oder Heu durch Silbenstruktur bedingte Allophone des gespannten Vokals /i/ sind (vgl. Morcienic 1958; Shannon 1984). Die Überlegung, dass -

42 Dass für das Wort Ion buslang keine Variante [jo:n], Jon' entstanden ist, mag daran liegen, dass [on] analog zu der Endung in Wörtern wie Proton, Neutron, Lepton als Suffix erkannt wird und somit eine eigene Silbifizierungsdomäne bildet. 
entgegen einer weit verbreiteten Auffassung - nur $/ \mathrm{u} /$ in Ansatzposition diachron als Frikativ reanalysiert worden ist, /i/ aber weiterhin in beiden Randpositionen erscheinen kann, basiert auf sprachübergreifender Evidenz für Sonoritätsabstufungen zwischen diesen Vokalen. Der Nutzen der sprachvergleichenden Perspektive zeigt sich weiter bei dem Erkennen von Silbifizierungsbeschränkungen, die eine Analyse der Gleitlaute als /i/-Allophone plausibel machen. So stützt etwa die Evidenz für eine Beschränkung gegen Ansätze mit mehr als zwei Phonemen im Französischen in Fällen wie bui[.ti] ème buitième, achtens' versus qua[.tri.]ème quatrième, viertens' die allophonische Analyse der Vokoide in En[..ti $]$ an versus Schlen[.dri.]an.

Selbst bei der Bewertung von Ausnahmen ist die sprachvergleichende Betrachtung nützlich. So zeigt sich nicht nur eine sprachübergreifende Präferenz für Regelverletzungen in Interjektionen; auch schriftsprachlich begründete Besonderheiten lassen sich leichter einordnen. Wenn etwa wortinitiales / i/ aufgrund der Entlehnung der Originalschreibweise durch das Graphem $<\mathrm{i}>$ repräsentiert ist, kann dieser Laut auch im Englischen unabhängig vom segmentalen Kontext als Nukleus organisiert und silbisch gesprochen werden (vgl. englisch [i.á..goun], Iago). Im Französischen hingegen bestehen solche schriftsprachlich begründeten Ausnahmen nicht: prävokalisches /i/ erscheint immer im Ansatz (vgl. französisch [jagó] ,Iago). Dass das Deutsche sich hier eher wie das Englische verhält, stimmt mit der insgesamt größeren Neigung zu lexikalisierter prosodischer Organisation von Phonemen in diesen beiden Sprachen, gegenüber dem Französischen, überein.

\section{Literatur}

Becker, Thomas (1998): Das Vokalsystem der deutschen Standardsprache. (= Arbeiten zur Sprachanalyse 32). Frankfurt a.M.

Brinkmann, Caren (i.Vorb.): Wortphonologische Experimente und Korpusanalysen. Mannheim.

Browman, Catherine P./Goldstein, Louis (1995): Gestural syllable position effects in American English. In: Bell-Berti, Fredericka/Raphael, Lawrence J. (Hg.): Producing speech: contemporary issues. New York, S. 19-33.

Bußmann, Hadumod (Hg.) (2002): Lexikon der Sprachwissenschaft. 3., aktual. u. erw. Aufl. Stuttgart.

Delattre, Pierre (1965): Comparing the phonetic features of English, French, German and Spanish: an interim report. Heidelberg.

Duden-Grammatik = Eisenberg, Peter (2009): Phonem und Graphem. In: Der Duden. Bd. 4: Die Grammatik. Hrsg. v.d. Dudenredaktion. 8., überarb. Aufl. Mannheim/ Wien/Zürich, S. 19-94. 
Eckert, Hartwig/Barry, William (2002): The phonetics and phonology of English pronunciation. Trier.

Essen, Otto von (1962): Grundbegriffe der Phonetik. Ein Repetitorium der Phonetik für Sprachheilpädagogen. (= Heilpädagogische Beiträge 8). Berlin.

Fouché, Pierre (1956): Traité de prononciation française. Paris.

Hall, Tracy Alan (1992): Syllable structure and syllable related processes in German. (= Linguistische Arbeiten 276). Tübingen.

Hall, Tracy Alan (2005): German glide formation as the interaction of faithfulness and markedness. In: Chicago Linguistic Society (CLS) 41, S. 81-94.

Harris, John/Gussmann, Edmund (2002): Word-final onsets. In: UCL Working Papers in Linguistics 14, S. 1-42.

Jakobson, Roman/Fant, Gunnar/Halle, Morris (1952): Preliminaries to speech analysis. The distinctive features and their correlates. 2. Aufl. (= Acoustics Laboratory, Massachusetts Institute of Technology, Technical Report 13). Cambridge, MA.

Jespersen, Otto (1913): Lehrbuch der Phonetik. 2. Aufl. Leipzig.

Kempelen, Wolfgang von (1791): Wolfgangs von Kempelen k. k. wirklichen Hofraths Mechanismus der menschlichen Sprache nebst der Beschreibung seiner sprechenden Maschine. Wien.

Kenyon, John S./Knott, Thomas A. (1953): A pronouncing dictionary of American English. Springfield, MA.

Kohler, Klaus (1977): Einführung in die Phonetik des Deutschen. Berlin.

Kohler, Klaus (1995): Einführung in die Phonetik des Deutschen. 2., neubearb. Aufl. Berlin.

Krech, Eva-Maria (1968): Sprechwissenschaftlich-phonetische Untersuchungen zum Gebrauch des Glottisschlageinsatzes in der allgemeinen deutschen Hochlautung. Basel.

Krech, Eva-Maria et al. (2009): Deutsches Aussprachewörterbuch. Berlin.

Lerond, Alain (1980): Dictionaire de la prononciation. Paris.

Liberman, Mark (1983): Phonetic representations. Paper presented at the Stanford Workshop on lexical phonology and Morphology.

Maas, Utz (1999): Phonologie. Einführung in die funktionale Phonetik des Deutschen. (= Studienbücher zur Linguistik 2). Wiesbaden.

Mangold, Max (2005): Der Duden. Bd. 6: Das Aussprachewörterbuch. Hrsg. v.d. Dudenredaktion. 6., überarb. u. aktual. Aufl. Mannheim u.a.

Meinhold, Gottfried/Stock, Eberhard (1982): Phonologie der deutschen Gegenwartssprache. 2., durchges. Aufl. Leipzig.

Morciniec, Norbert (1958): Zur phonologischen Wertung der deutschen Affrikaten und Diphthonge. In: Zeitschrift für Phonetik und allgemeine Sprachwissenschaft 11, S. 49-66. 
Moulton, William G. (1962): The sounds of English and German. Chicago.

Mücke, Doris (1998): Der j-Laut im Deutschen: Normierung und Gebrauchsnorm. In: Kröger, Bernd J./Riek, Christine/Sachse, Georg (Hg.): Festschrift Georg Heike. (= Forum phoneticum 66). Frankfurt a.M., S. 97-113.

Muthmann, Gustav (1996): Phonologisches Wörterbuch der deutschen Sprache. (= Reihe Germanistische Linguistik 163). Tübingen.

Philipp, Marthe (1974): Phonologie des Deutschen. Stuttgart.

Pompino-Marschall, Bernd (1995): Einführung in die Phonetik. Berlin.

Raffelsiefen, Renate (einger.): Sonority thresholds in English, French and German. In: Phonology.

Ramers, Karl-Heinz/Vater, Heinz (1995): Einführung in die Phonologie. 4., überarb. Aufl. (= Kölner linguistische Arbeiten, Germanistik 16). Hürth.

Schiller, Niels O./Mooshammer, Christine (1995): The character of /r/-sounds: Articulatory evidence for different reduction processes with special reference to German. In: Elenius, Kjell/Branderud, Peter (Hg.): Proceedings of the XIIIth International Congress of Phonetic Sciences. Bd. 3. Stockholm, S. 452-455.

Shannon, Thomas F (1984): The syllable as a descriptive and explanatory parameter in German. In: Dressler, Wolfgang U./Pfeiffer, Oskar E./Rennison, John R. (Hg.): Discussion papers for the Fifth International Phonology Meeting. (= Wiener Linguistische Gazette 3). Wien, S. 235-239.

Siebs, Theodor (1905): Deutsche Hochsprache: Bühnenaussprache. 3. Aufl. Berlin.

Siebs, Theodor (1957): Deutsche Hochsprache: Bühnenaussprache. 16., völlig neubearb. Aufl. Berlin.

Tranel, Bernard (1987): The sounds of French: an introduction. Cambridge.

Trubetzkoy, Nikolaj S. (1958 [1938]): Grundzüge der Phonologie. 2. Aufl. Göttingen. [1. Aufl. 1938].

Vennemann, Theo (1982): Zur Silbenstruktur der deutschen Standardsprache. In: Vennemann, Theo (Hg.): Silben, Segmente, Akzente. (= Linguistische Arbeiten 126). Tübingen, S. 261-305.

Vennemann, Theo (1988): Preference laws for syllable structure and the explanation of sound change: with special reference to German, Germanic, Italian, and Latin. Berlin.

Vennemann, Theo (1994): Universelle Nuklearphonologie mit epiphänomenaler Silbenstruktur. In: Ramers, Karl Heinz/Vater, Heinz/Wode, Henning (Hg.): Universale phonologische Strukturen und Prozesse. (= Linguistische Arbeiten 310). Tübingen, S. 7-54.

Viëtor, Wilhelm (1921): Deutsches Ausprachewörterbuch. 3. Aufl. Leipzig.

Warnant, Léon (1987): Dictionnaire de la prononciation française dans sa norme actuelle. 4. Aufl. Paris u.a. 
Wells, John C. (2000): Longman pronunciation dictionary. Harlow.

Wiese, Richard (1996): The phonology of German. Oxford.

Wurzel, Wolfgang U. (1970): Studien zur deutschen Lautstruktur. (= Studia grammatica 8). Berlin.

Zifonun, Gisela/Hoffmann, Ludger/Strecker, Bruno (1997): Grammatik der deutschen Sprache. 3 Bde. (= Schriften des Instituts für Deutsche Sprache 7). Berlin u.a. 
